\title{
Test Program for High Efficiency Gas Turbine Exhaust Diffuser
}

\section{SBIR Grant DE-FG02-07ER84680}

Consultants in Engineering Acoustics

Thomas R. Norris

December 31, 2009 


\section{Executive Summary}

This SBIR project relates to testing a way to improve flow efficiency around struts in turbine exhausts, and thus the turbine efficiency as well. This Phase 1 effort demonstrated the technical viability of "strutlets", which are a novel flow-improving concept to be applied to fossil-fueled power plants with gas turbines. By improving gas turbine exhaust diffuser flow, strutlets can reduce backpressure, improve fuel efficiency and power, and reduce the emission of greenhouse gases. The strutlets were tested in a physical scale model of a gas turbine exhaust diffuser with a straight, annular flow passage, which is the most common configuration for large turbine diffusers at power plants. The long-term goal of reduced backpressure would, if achieved, improve fuel efficiency and power by 0.5 percent, averaged over US gas turbine power plants. Greenhouse gas emission would be reduced by the same amount. At the end of the Phase I modeling and testing, the strutlets did improve flow attachment to struts, but did not achieve the stated efficiency goal. For the technology to be practical in commercial applications, the added aerodynamic drag resulting from the strutlets would need to be reduced. Independently sponsored follow-on work developed a lower drag strutlet, and confirmed through model testing that the lower-drag design could meet the project goals for a simple-cycle power plant, and most of the goals for a combined cycle. Using reduced-drag strutlets in combination with other flow improvements, low-frequency pulsation and sound were also reduced by $5 \mathrm{~dB}$ or more.

To establish baseline diffuser performance, CIEA used raw flow data from an existing full-scale turbine, a Siemens-Westinghouse $501 \mathrm{G}$ operating at 3600 RPM. This is a large turbine, in the 250-MW class. Qualitative probing with a pitot tube downstream of struts showed modest but persistent slow flow zones near the hub, rather than the desired narrow strut wakes. Narrow strut wakes would indicate efficient flow, while the slow flow zones indicate flow losses. Near the downstream end of the diffuser, flow was sometimes locally intermittent, which might be described as repetitive "puffs." Overall diffuser efficiency was reasonably good at a pressure recovery of 0.68 to 0.72 , with one data point at 0.75 at a reduced power setting.

A scale model was assembled, and the flow around the first set of struts evaluated. A partially completed physical scale model of the exhaust diffuser of a $501 \mathrm{G}$ combustion turbine was used, because it was available; and, very importantly, it had typical realworld flow issues. The scale was 0.376 to 1 . The model proved to have flow patterns similar to those of the full-size turbine. However, there were differences--the model had significant stalls at all strut intersections at both the hub and the rim, while the actual turbine showed slow flow zones just at the hub.

Four styles of "strutlets" were attached one at a time to one strut. As part of an initial screening test, tassels were taped to the strut to see if the flow would stay attached. Attached flow tends to be efficient; stalled flow does not. The baseline was mostly in stall (detached) flow. All four strutlets succeeded in keeping flow attached. However, so 
did the control, a simple airfoil spaced a distance away in the manner of a leading edge slat on an airplane wing. The use of strutlets did appear to result in a stronger attachment, so they were judged as promising for application when a major flow improvement might be required. An example is when there is incident flow with a high angle of attack, such as usually occurs when a turbine operates at reduced power. A series of total pressure traverses, however, revealed that the strutlets also produced a strong wake. The principal investigator judged that this wake would, at its observed strength, counter the intended benefit. The conclusion of Phase I work is that the strutlets could promote flow attachment, but would have to be redesigned for reduced drag. Extending the flow modifiers into the remainder of the diffuser was always planned, in combination with other flow-modifying techniques.

After the Phase 1 technical work was finished, independently sponsored research pursued refinement of the design. A low-drag strutlet was designed and tested. When this lower-drag strutlet was installed in the model diffuser, diffuser performance improved sufficiently to meet the 0.5 percent power and heat rate improvement goal, for simple-cycle power plants, and most of the goals for combined cycles. Perhaps as importantly, low frequency sound or pulsation in the diffuser was reduced by more than $5 \mathrm{~dB}$, compared to the baseline diffuser. This appears likely to translate into a similar reduction in exhaust outlet noise, a significant environmental advantage at many power plant sites. The results of this post Phase 1 effort appear in Appendix A.

\section{Nomenclature}

Pt Total Pressure, in Inches of Water Column

Pv Velocity Pressure, " " " "

Ps Static Pressure, " "

$\mathrm{Cp}$ Diffuser Coefficient of Pressure Recovery

Cpi Ideal Coefficient of Pressure Recovery

w.c. Water Column, a measure of pressure 


\section{APPROACH}

\section{Scope of Investigation}

This Phase I SBIR project evaluated the technical viability of an innovative flowimproving concept in a model of a power generation combustion turbine exhaust. More specifically, this concept is applicable to gas turbines that have struts in their exhaust diffusers, which includes most turbines used in the power industry. Improved flow there could result in more power, better heat rate, less fuel use, and less greenhouse gas emission. Diffuser noise and vibration could also be reduced.

This project was not selected to receive a Phase II DOE grant. However, the grantee chose to pursue the Phase II scale modeling work independently, based on the mixed results of Phase 1, and a hunch. The original technical goal of Phase II was to refine the flow-improving concept, and to demonstrate its role in the rest of the diffuser. To support the required SBIR commercialization plan, the flow-improving technology would then be demonstrated in an existing power plant gas turbine exhaust. CIEA did apply the flowmodification technology to the next sections of the diffuser. Modeling and testing have been completed and the commercial demonstration is in contract negotiations.

The main body of this report describes the Phase I work. For completeness and to avoid possibly leading the reader to a wrong conclusion, Appendix $A$ is a summary of the most important follow-on, non-DOE, independent research performance results.

The exhaust diffuser is an aerodynamically shaped duct section located just downstream of the last stage turbine wheel. Excellent flow through the diffuser, especially around the struts, will improve turbine power and efficiency. The long-term goal is to improve the power and efficiency by one-half percent, averaged over the U.S. turbine fleet. The goal of this Phase I project, the first step, is to demonstrate smooth, efficient flow around the struts in a model turbine, in the presence of realistic turbine flow swirl, radial velocity profile, and various pressure gradients.

The general concept is shown in Figure 1.

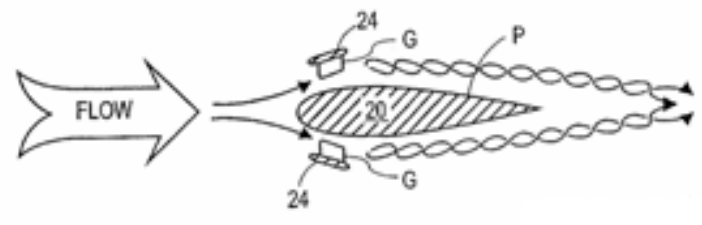

Figure 1. General Concept - Cross Section

As shown in Figure 1, Structural Strut 20 is equipped with Strutlets 24 and Vanelets G. Intended use of strutlets is in turbomachine exhausts [CIEA Patent Application]. The concept is to mount vortex-generating elements for diffuser struts and walls in free stream flow, rather than in the boundary layer The strutlet consists of an airfoil 
positioned near the strut. Attached to the airfoil are vortex-generating devices, which in combination, cause flow to stay "attached" to the strut.

The smooth attached flow, or lack of attached flow, was determined using a physical scale model of an actual power plant gas turbine exhaust diffuser. Discussion of the work is organized into sections on making and testing the model, baseline testing, testing with added "strutlets,"and data analysis.

The experiment design philosophy emphasizes quickly trying variations of an unusual concept, rather than the experimental rigor appropriate for an in-depth evaluation or precision refinement of one existing design. Consequently, the models are not precise, but are nonetheless adequate for their intended purpose. For example, a 1/8 inch high step at junctions in the five-foot-diameter diffuser was judged acceptable provided that the step was not varied from test to test. CIEA also chose to use a larger model than is used in most studies, simply because a large model setup was available in its lab, and was almost ready to install on the test rig fan.

\section{Phase I Objectives}

Objective 1: Choose a turbine to model and define its geometry. A large GE or Siemens-Westinghouse frame machine with straight exhaust path is slightly preferred, but a right angle bend exhaust is also very acceptable.

An operating power plant was chosen, on which CIEA had begun other diffuser work. The turbine is a Siemens-Westinghouse 501G, rated at $253 \mathrm{MW}$. The owner agreed for CIEA to use raw data, but requested that the power plant not be identified. Thus, the raw data is a contribution to this SBIR project. The exhaust diffuser and interior geometry was measured and drawings were prepared by an outside engineering firm, shown on Figure 2. 


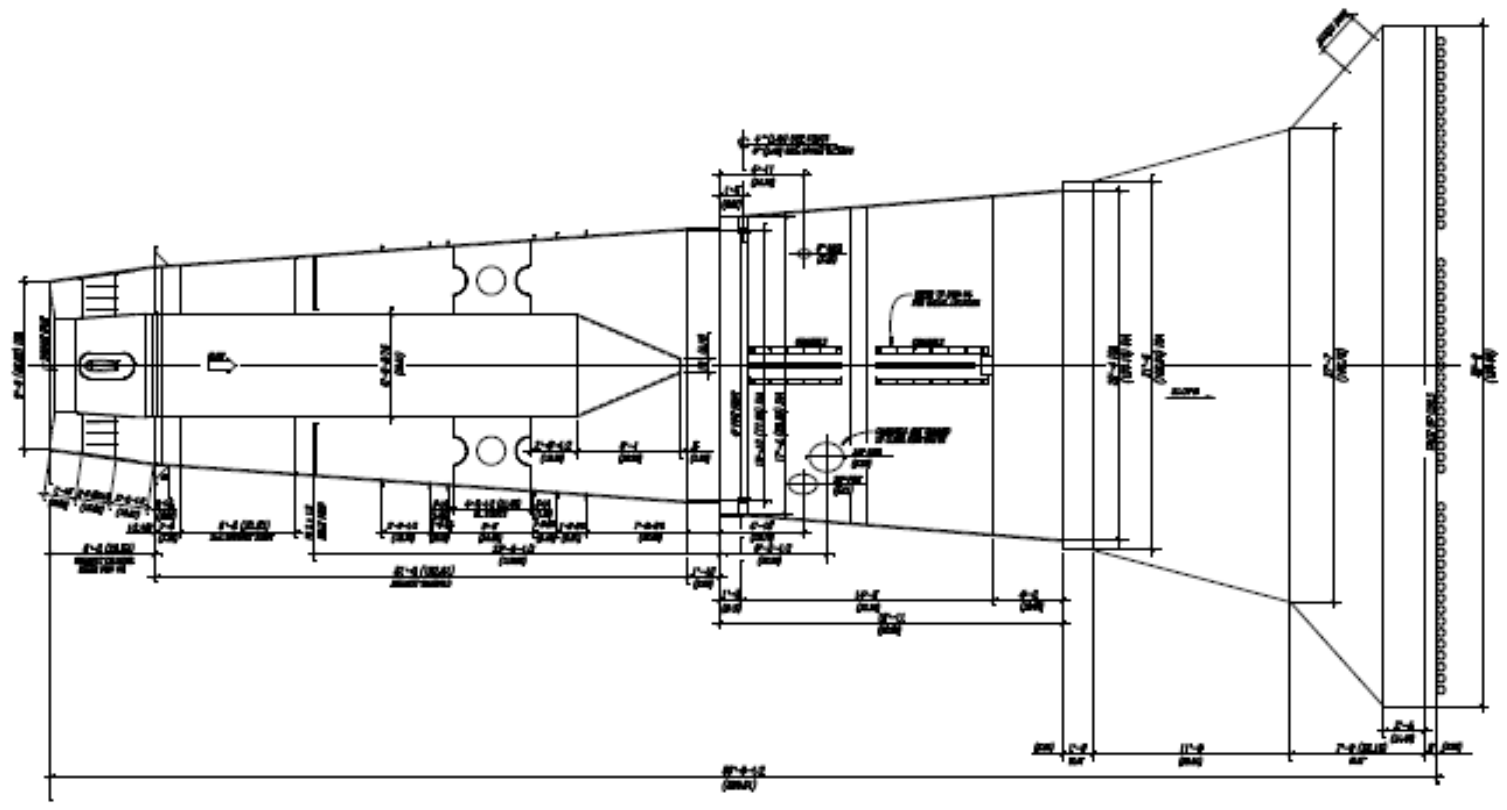

PLAN VIEW,

Figure 2. Exhaust Diffuser and Interior Geometry. The Phase 1 Project Tests Were Conducted in the Inlet TRF Section at Far Left with the Struts.

An inspection found one repaired crack at the trailing edge of an exhaust cylinder strut, which is not unusual for large turbines. The crack had not spread since the repair was made. Several cracks were found in the diffuser proper, all associated with welds between plates or at plate-to-flange joints. This pattern has been seen in other gas turbine exhaust diffusers.

As to the diffuser geometry, distances are measured from the diffuser inlet plane, and cone taper angles are the "half-angles" measured between the diffuser axis and the surface under consideration. Overall, the diffuser length is 55 feet, the inlet diameter 9.75 feet, and the "area ratio" (AR) is about 6.18. The area ratio is the outlet flow area divided by the inlet area. This DOE Phase I study involves primarily the first strut section, at the far left side of Figure 2, which is 6 feet long with an AR of 1.46.

In this report, axial location is denoted by "station," expressed in feet from the turbine wheel. (When referring to the scale model, the axial station is in inches, again measured downstream from where the turbine wheel would be.)

The $501 \mathrm{G}$ exhaust diffuser shape is common to most large turbine diffusers in the power industry, with a roughly cylindrical center body and a diverging outer cone, forming an annular flow passage with a flow area that grows in the direction of flow. The 
angle of divergence is near 5 degrees to the diffuser axis. There are three sections, totaling 55 feet in length.

The first section, at far left on Figure 2, is 6 feet long (station 0 to 6 ). This section is variously termed "exhaust cylinder" or "turbine rear frame" (TRF), and has the six struts which support the turbine's rear main bearing. The term TRF is used in this report. Figure 3 shows a view of the $501 \mathrm{G}$ TRF, looking into flow.

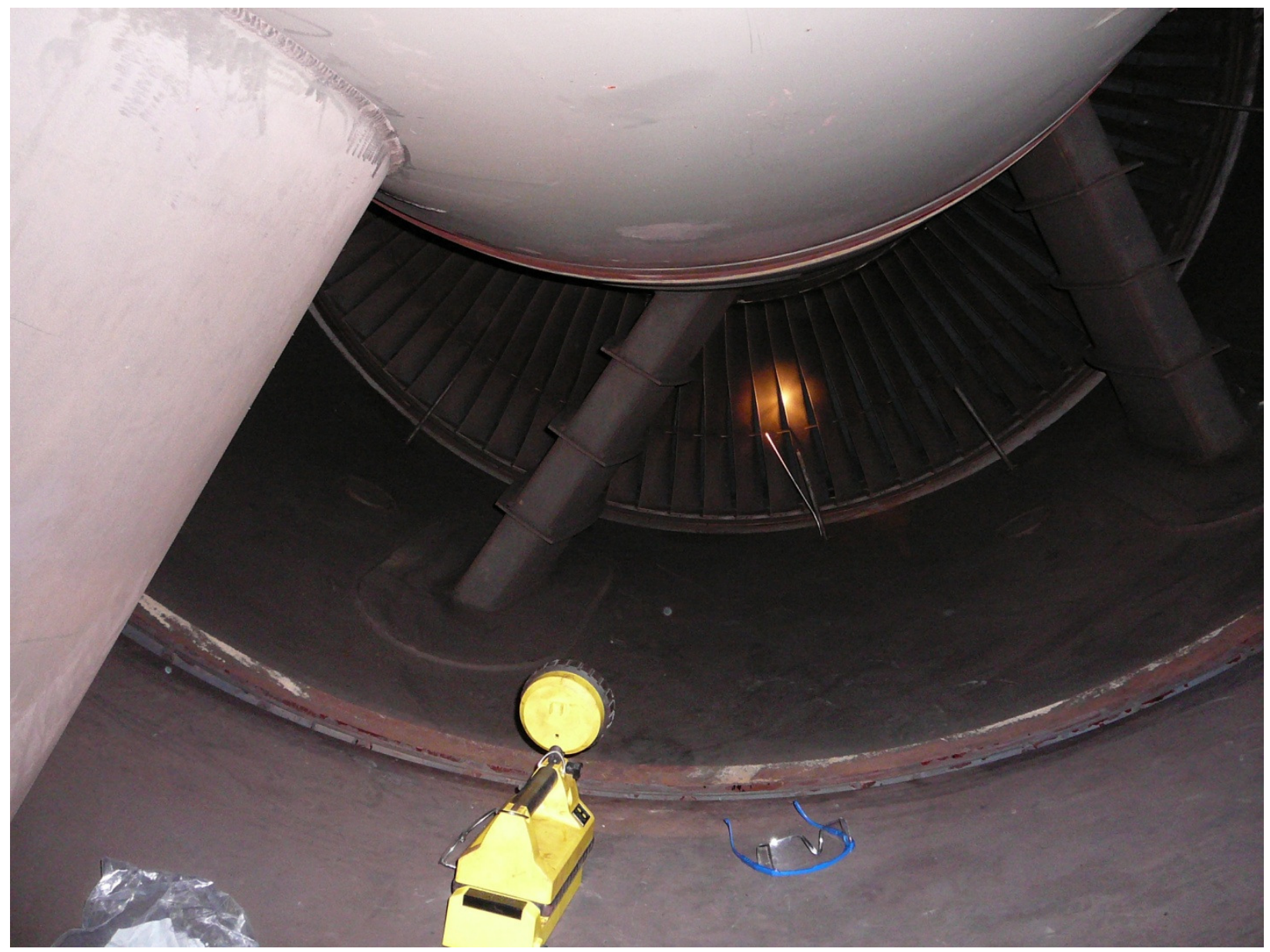

Figure 3. Turbine Wheel, Looking into the Diffuser

The hub (center body) has a small growth taper for its first 0.75 feet, followed by a substantially cylindrical shape extending far downstream to station 39 , which is 70 percent of the diffuser total length. The outer shell expands at 8 degrees for the initial 6foot-long section. This diffuser system has three groups of struts; only the first is investigated in this study, but the others are described as well. The first or TRF strut group, shown in Figure 3, consists of six struts that support the turbine rear main bearing, located within the exhaust cylinder, which is the first diffuser section encountered by the exhaust flow. These struts are "set" to zero swirl angle, so that with zero swirl flow coming from the turbine last stage, the aerodynamic angle of attack is zero degrees, and the smoothest flow would be expected. The strut is a streamlined symmetric shape, but has a blunt "boat tail" trailing edge shape. An ingenious feature is 
that the six struts which support the turbine shaft and rear bearing are canted 30 degrees from radial, so that strut thermal expansion can result in a slight rotation of the rear main bearing housing, rather than creating high stress levels in the struts. Thus, these struts can function without a cooling air fan, despite being in a red hot environment. However, the acute (approximately 60 degree) angle thus created at the strut-to-wall intersections is expected to be a potential source of drag, and should receive attention in a final design.

The second diffuser section extends from stations 6 to 39. The center body terminates at approximately 38 feet. There are two more strut sets; the first a pair having a quite large tear-drop shape are about 7.5 feet from the inle; and the second being singlethickness steel, at about station 30. Again, both strut sets are aligned for flow with zero swirl. The second strut set supports the first section of the center body, and also houses the rear main bearing's oil supply piping. Remarkably, these two struts are large enough to be equipped with internal ladders for human access to the center hub and bearing. The third strut set consists of four, rather thin, contoured plates, and supports the aft portion of the center body.

The third diffuser cone section extends from station 39 to 55 feet. It starts with a roughly one foot high step expansion, and then expands at about a 5-degree angle to its end. This was scale modeled only to this end point, station 55 . Beyond this, the duct is abruptly rectangular, expands rapidly, and terminates at a HRSG inlet.

An important measure of diffuser performance is the coefficient of pressure recovery, denoted $\mathrm{Cp}$. This is the ratio of inlet kinetic flow energy converted to useful negative pressure at the diffuser inlet. A Cp of 1.00 indicates that all of the kinetic energy is recovered, and 0 indicates that none is recovered. In the ideal case, $C p i=1-1 /\left(A R^{\star} A R\right)$. At the end of the first diffuser section, at station $6, A R=1.46$ and the Cpi is 0.53 . Thus, over half of the pressure recovery would be ideally in the first 6 feet, or $11 \%$ of the diffuser length. Not surprisingly, there is no substitute for excellent aerodynamic performance here.

Several details must be noted, because they may reduce performance. These are the types of additions that might enter a turbine design sometime after the basic, usually excellent aerodynamic design is complete. First, cooling air is admitted at the diffuser inlet plane, both at the hub and at the rim. There is a small step expansion at the rim that may enhance this cooling flow. There are also several small welded steps in the hub and rim at several locations. At the first section outlet, station 6 , there is a circumferential slot at the rim. The struts each have three fins on each side. These may be stiffeners, but also create wakes.

In the second diffuser section, there are a few longitudinal and circumferential strips welded to the diffuser interior walls, possibly repairs, added seals, or braces. There is the step expansion at 39 feet into the diffuser, or at nearly $71 \%$ of the diffuser length measured from the inlet. 
The third diffuser section includes numerous longitudinal fins that appear to be part of the duct liner retention structure. There are also four cross-pipes, perpendicular to flow, in the third section.

The proposed auxiliary strutlets with vortex-generating features could be mounted on the six TRF struts, adjacent to the strut leading edges. Some flex would be needed for an actual turbine installation because of thermal expansion. Providing such flex was judged feasible, but is not otherwise a concern at the scale model design stage.

Objective 2: Adapt model to the chosen turbine and validate reconfigured turbine flow model. Consult with subject-matter experts. The diffuser consultant assisted in fan system modification design; as it turns out, he built and "trouble-shot" a similar system many decades ago. The industry consultant reviewed strut design for thermal expansion compliance, history of cracking, and other issues that would affect attachment of new vanes.

The model fan outlet swirl guide vanes were found to have stalls on the pressure (concave) sides, due to the simple single-thickness airfoil shape. These stalls allowed radial transfer of stalled gas inward, toward the hub, where in some areas it accumulated, resulting in a zone of slow flow. Converting to a conventional, thick airfoil shape eliminated these stalls and consequently eliminated the large slow-flow zones near the hub. To improve inlet flow, the motor was remounted farther from the fan and the shaft was extended. To reduce bearing stresses, a second flexible coupling was installed, and the fan lower frame "l" beams were supplemented with 6-inch heavy wall square steel tubing. These square tubes were supported from the floor on eight 1-inchdiameter jack screws.

Measurements on the operating turbine at full power showed that from near the hub to near the rim, the velocity pressure was substantially constant at about 80 inches water column. Measurements proved difficult to make, and within 4 inches of the hub and rim, measurements were left incomplete because an instrument pressure tube bundle failed. It was not possible to repeat the tests due to power plant shutdown schedule limitations. At maximum power, $230 \mathrm{MW}$ during tests, swirl at 8 inches from the rim was measured at -10 degrees. (The negative angle denotes swirl opposite the rotation of the last stage turbine disk.) At 4 inches from the hub, swirl was +20 degrees, a value with considerable uncertainty because of a nearby strut. The overall $\mathrm{Cp}$, coefficient of pressure recovery, was at most power settings 0.68 to 0.72 , about at the lower end of the range expected. One $\mathrm{Cp}$ data point was 0.75 , measured at slightly reduced power, and was the best data point recorded. The diffuser pressure performance was measured at 10 inches downstream of the turbine wheel using a very rugged probe with the tip similar to that of an "S" pitot tube combined with a three-hole probe.

There are some other significant uncertainties in these data. These include turbulence close to the turbine wheel affecting the probe, the pressure fields near struts, and an unknown static pressure rise in the first 10 inches from the turbine wheel. Flow in this 
first 10 inches includes a step expansion, two air inlet slots for hub and rim cooling flow, and presumed locally strong swirl from the blade tips.

The scale model fan inlet guide vanes (IGVs) allow adjusting the radial velocity profile, and were set for a uniform velocity pressure from hub to rim. The model fan outlet guide vanes (OGVs) were reset to provide 10 degrees swirl at the rim, and 20 degrees at the hub. The swirl was set in the same direction, rather than in opposite directions, a compromise thought to not affect the specific evaluation of the strutlet flow improvement devices under consideration.

Objective 3: Design, make, install, and test new baseline model. Existing diffuser was used, with modification. The diffuser consultant observed the installation.

The initial intent was to use an existing simple scale model. However, a more complex $501 \mathrm{G}$ model, then partially completed, became available, and was selected. The struts were known to have some real-world flow issues involving swirling inlet flow and significant wakes near the hub. This model also has the potential of being used to prove a complete diffuser modification kit, and could be used to measure entire diffuser $\mathrm{Cp}$ values. This would be a prelude to commercialization. (Commercial application is a required goal of any SBIR project.)

The model was completed and assembled. Figures $4 \mathrm{~A}$ and $\mathrm{B}$ show the model TRF during assembly.

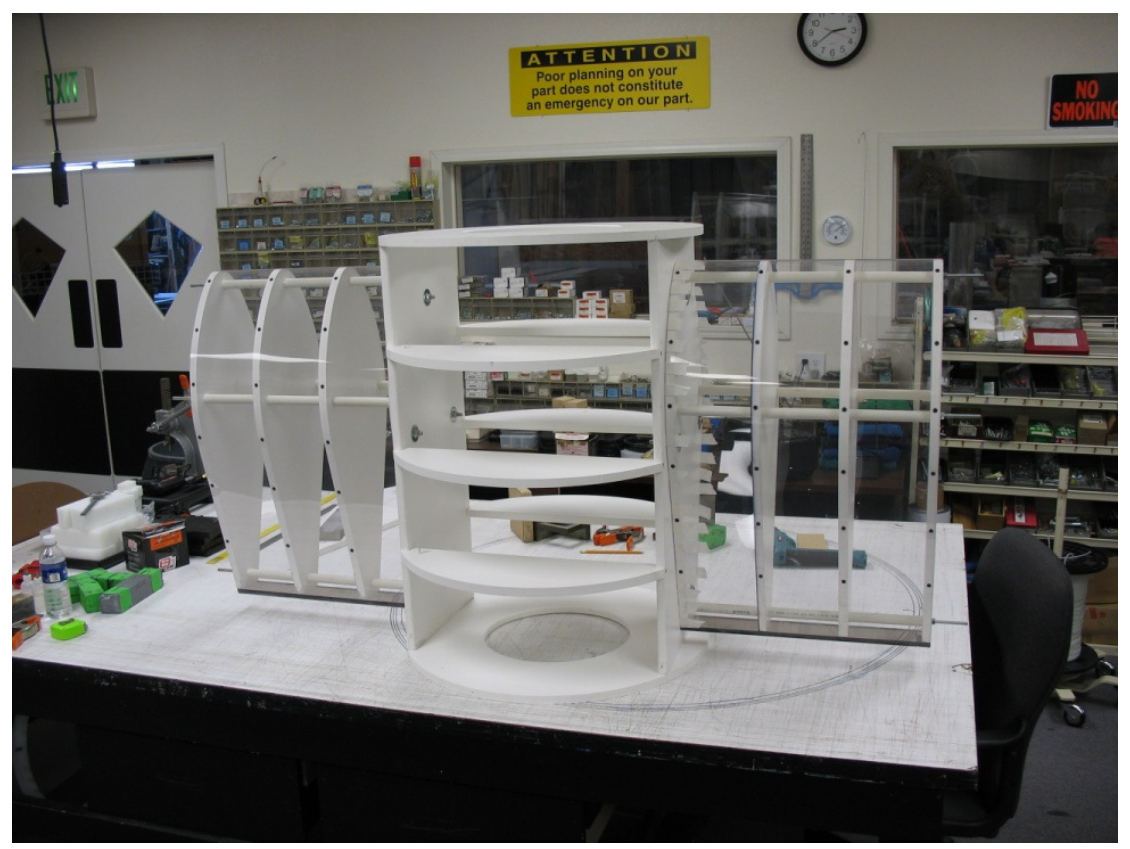

Figure $4 \mathrm{~A}$ 


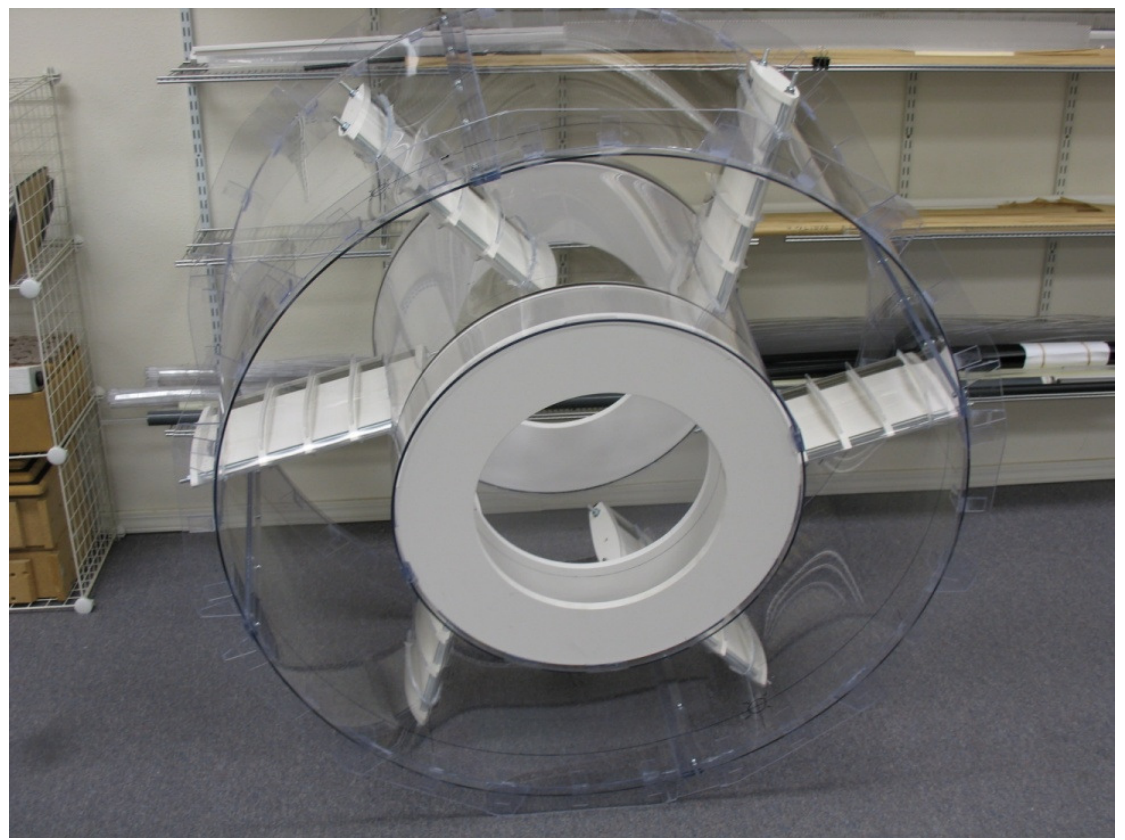

Figure 4B

Figure 4. Views of Scale Model During Fabrication

A second set of OGVs were added. Figure 5 and 6 show the overall model.

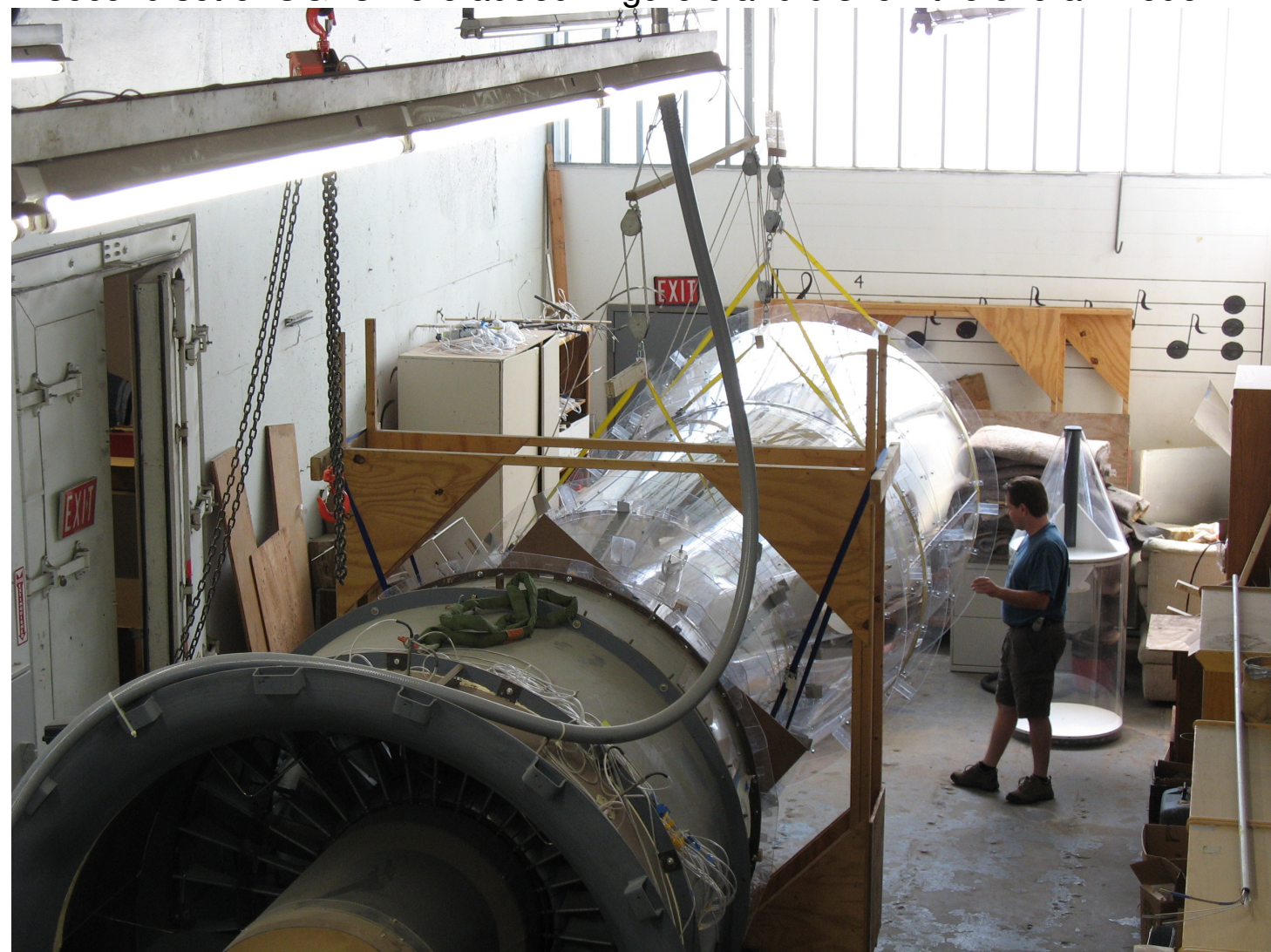

Figure 5. External View of Overall Model 


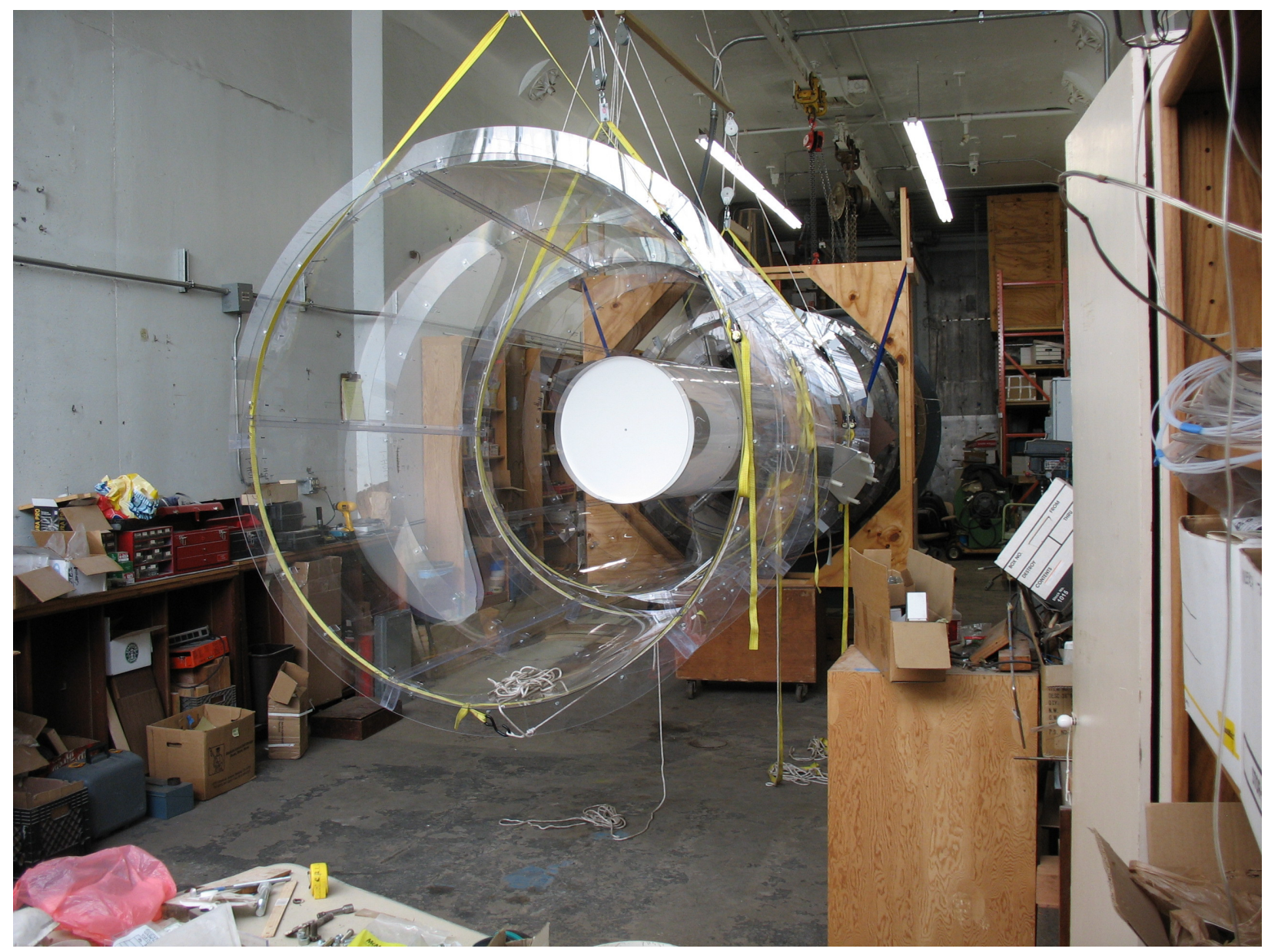

Figure 6. View of Model from Outlet End. Center Cone and Last Diffuser Section Are Not Yet Attached

The total model diffuser length is 20.5 feet (station 245), at the scale factor of 0.376 to 1.

Objective 4: Design and test trial vane sets for struts. Visits by each consultant for comments on airfoil shapes, practicality, market acceptance, and performance.

Although developing an algorithm was originally part of this objective, it was decided that the algorithm would not be productive, because it was already apparent that the drag would be high. Also, the scale model would give the same result as the algorithm, but do so very quickly, and with less uncertainty.

Objective 5: Prepare [final] report documenting results. State clearly if technical goals were met, and if not, why not. State an opinion on commercial viability, possibly after further Phase II optimization. This is the final report. 


\section{RESULTS AND DISCUSSION}

This section discusses the test procedures, including flow conditioning, instrumentation.

\section{Measurement and Conditioning of Inlet Flow}

Inlet flow measurement and conditioning is one of the most important, and at first easily overlooked, items in a diffuser model test. In the Phase I work, the goal was simply to improve flow around struts, as evidenced by lack of stalls.

With respect to strut flow, the important inlet flow parameter is swirl angle, provided that the boundary layers on the rim and hub were reasonably thin so that flow would be stable and realistic. In Phase I, there were problems with corner stalls.

The test setup consists of a large fan, a transition section, a flow measurement section, and, finally, the test diffuser. The annular inlet and measurement section for this work is described next. All diameters are for the hub or rim surface in contact with flow. The 72inch fan is rated at above $125 \mathrm{lbs} /$ second mass flow in air. The flow annulus then decreases to 60 inches diameter in a transition that also houses a set of swirl vanes. The next duct section reduces the diameter further to 44 inches. A second set of swirl vanes at the end of the transition provides a fine adjustment of swirl, and generates some turbulence. A constant area measurement section is formed by a pair of cylinders to form an annular flow space, 44 inches outside diameter, 27 inches inside diameter, and 30 inches long. This left a little over two annulus widths for measurement purposes. Static pressure was determined at station -10, slightly over one annulus width upstream of the diffuser inlet, and downstream of the smaller swirl vanes. Static pressure was sensed by equally spaced 0.125 inch diameter holes in the measurement annulus walls: eight holes in the outer wall, and four in the inner wall. Total pressure was measured upstream of this section where the flow area is larger, using eight small metal tubes aimed directly into the flow.

The Reynolds number at the diffuser inlet, using diffuser inlet diameter is 1.2 million. Based on the TRF strut chord or annulus width at the diffuser inlet, it is about 350,000. The latter is in a range where due care must be taken to "trip" boundary layers, but flow is otherwise fully turbulent. This "tripping" was done with tape strips, or by leaving tiny steps at the trailing edge of strut nose pieces.

\section{Instrumentation}

The data of most interest are flow patterns and stalls around the struts on their suction sides. Other data are model inlet and outlet flow traverses, and traverses of flow on lines out from and perpendicular to from the suction side of struts. (The pressure side of struts, receiving incident swirling flow, rarely show separations so are not instrumented.)

Pressures were measured with one of two systems. The first is a 16 channel electronic pressure system, Scanivalve Model 3018, connected to a Toshiba laptop computer 
equipped with Scanivalve DSA Link software. Resolution is 0.0025 inches w. c. on channels $1-8$, and 0.005 inches on channels 9 to 16 . The unit was calibrated by Scanivalve Corporation within six months of these tests.

The second pressure measurement equipment is two analog differential pressured gauges of modest accuracy, specifically Dwyer Magnehelic gauges, one with a range of 0 to 0.5 inches and the other, 0 to 2.0 inch water column (w.c.). These are rugged meters, with rated accuracy at 3 percent of full scale. When readings were compared, we could see no differences between the Scanivalve and the two Dwyer meters, within the accuracy that could be read.

Diffuser inlet flow total pressure, (Pt) was traversed with the diffuser disconnected. Thus, near the rim, the total pressure is almost identical with velocity pressure (Pv.) This is because the rim static pressure (Ps) is very close to room ambient pressure. However, at the hub, the static pressure is significantly less than room pressure, mostly due to swirl, so the Pt values are less than the absolute magnitude of the Pv values. Regardless, for simplicity and to expedite work, Pt values are used throughout most of this report. The traverse probes are "rakes" of fourteen 0.125 inch outside diameter stainless steel tubes. The tubes are supported on a stick that pivots at the diffuser axis. The tubes are aimed directly into a (presumed) 15 degree swirl flow. These tubes and actual flow angles were thus aligned within 5 degrees, resulting in insignificant systematic error (under 2 percent) due to the flow and tubes not being exactly aligned. Angular spacing of the measurements around the model rim is every 2 degrees, and radial probe spacing is approximately an inch mid-annulus, decreasing to 0.25 inch for the outermost and innermost five probe positions. The probe could be positioned at two radii, to create overlapping sweeps, resulting in a higher resolution in the flow contour "picture."

The model diffuser TRF outlet flow, at station 27 inches, was measured in the same way and with the same probe set, but the tubes were spaced farther apart to account for the larger flow annulus diameters.

Total pressure traverses extending out from the struts were measured with the a single 0.25 inch copper tube aimed into the flow, and positioned by hand braced against a ruler extending across the flow. This positioning accuracy is estimated at within about a one-eighth inch at positions near the strut, and at a quarter inch more than three inches out. This was judged acceptable accuracy for the intended purpose of making initial screening comparisons between strutlets, and discovering any large stalls or unusual strutlet wakes.

\section{Data Discussion and Results}

Figure 7 is a total pressure flow traverse at the diffuser model diffuser inlet position, which is axial "station" 0 , with the flow exhausting into the room. 


\section{Revised Baseline Diffuser Inlet Velocity Scan Flow Data}

alpha= kinetic energy flux profile factor $=1.04$
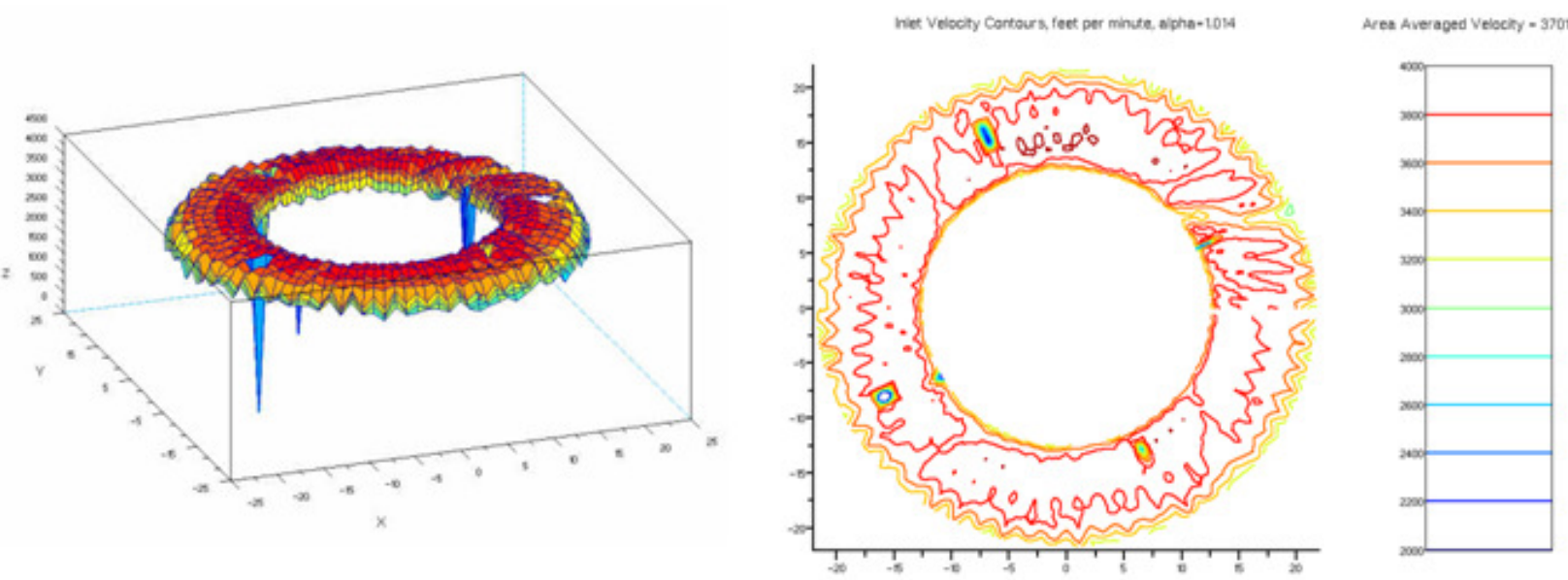

Figure 7. Revised Baseline Diffuser Inlet Velocity Scan Flow Data

Axial position or "station" is measured from the model diffuser inlet (the turbine wheel trailing edge plane on the real turbine) with negative numbers denoting positions farther upstream, in the inlet flow measurement duct. The overall flow is seen to be substantially uniform, excepting the wakes of four thin rods that support the hub, and a wake at the two o'clock position from an upstream data tube bundle. These rods were less than an inch upstream of the probe tips, so gave a sharp, readily seen pressure dip if the probe was in the wake. The serrated pattern visible on both views is caused by wakes from the second set of 32 fan OGVs that adjust the swirl.

Turbulence, as judged by tassel motion, is substantial, judged to be a little greater than is seen in fully developed flow in a pipe. Tassel motions were large, suggesting that the turbulence scale was large. However, turbulence was not quantitatively measured.

Figure 8 shows a total pressure traverse of diffuser outlet flow from the TRF (exhaust cylinder, model station 27) in the baseline configuration. 

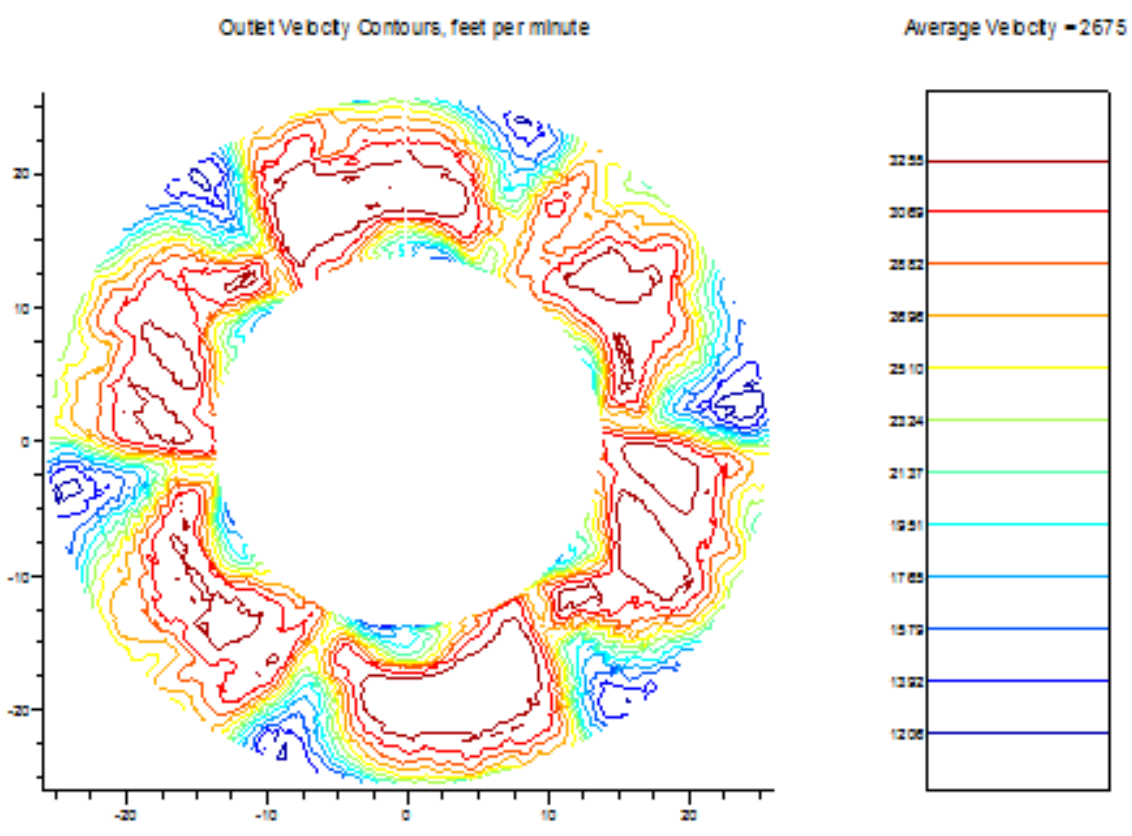

Figure 8. Velocity Scan of Exit Flow

(This is the diffuser section with the six struts, 6 feet long on the full scale turbine.) The effects form the six struts dominate the profile. On the suction side of each strut, at the rim, is a large low velocity zone, roughly triangular in shape. A smaller, similar shape appears at the hub, but displaced slightly away from the strut. Both low-velocity zones are typical phenomena associated with struts in a test diffuser. In contrast, the pressure side of each strut shows thin boundary layers and no flow separations.

These two low-flow zones near the suction side of each strut are significant; their presence excludes good flow around the struts themselves. Also, these 12 large lowenergy zones will promote weak flow zones farther downstream in the diffuser. So, achieving more efficient strut flow must include eliminating these two large low-flow zones near each strut. Indeed, in several informal experiments with slats and strutlet vortex generators, even if good strut flow was achieved on the strut itself, these other low-energy zones simply enlarged downstream, and the $\mathrm{Cp}$ with the entire diffuser attached did not significantly improve.

In view of the corner stalls, for the strutlet tests, a corner flow device was installed and left in place. This was basically a cambered strut placed between the rim and the strut, approximately perpendicular to a line that bisects the corner. It reduced the corner stall effect on the strut itself, but certainly did not eliminate the corner stall. This would not have application in an actual turbine.

Figure 9 shows a trial method of eliminating the low-flow zones, which is to install unusually large fillets at the strut-rim and strut hub junctions. This was successful in eliminating the corner stalls and low-energy zones. 


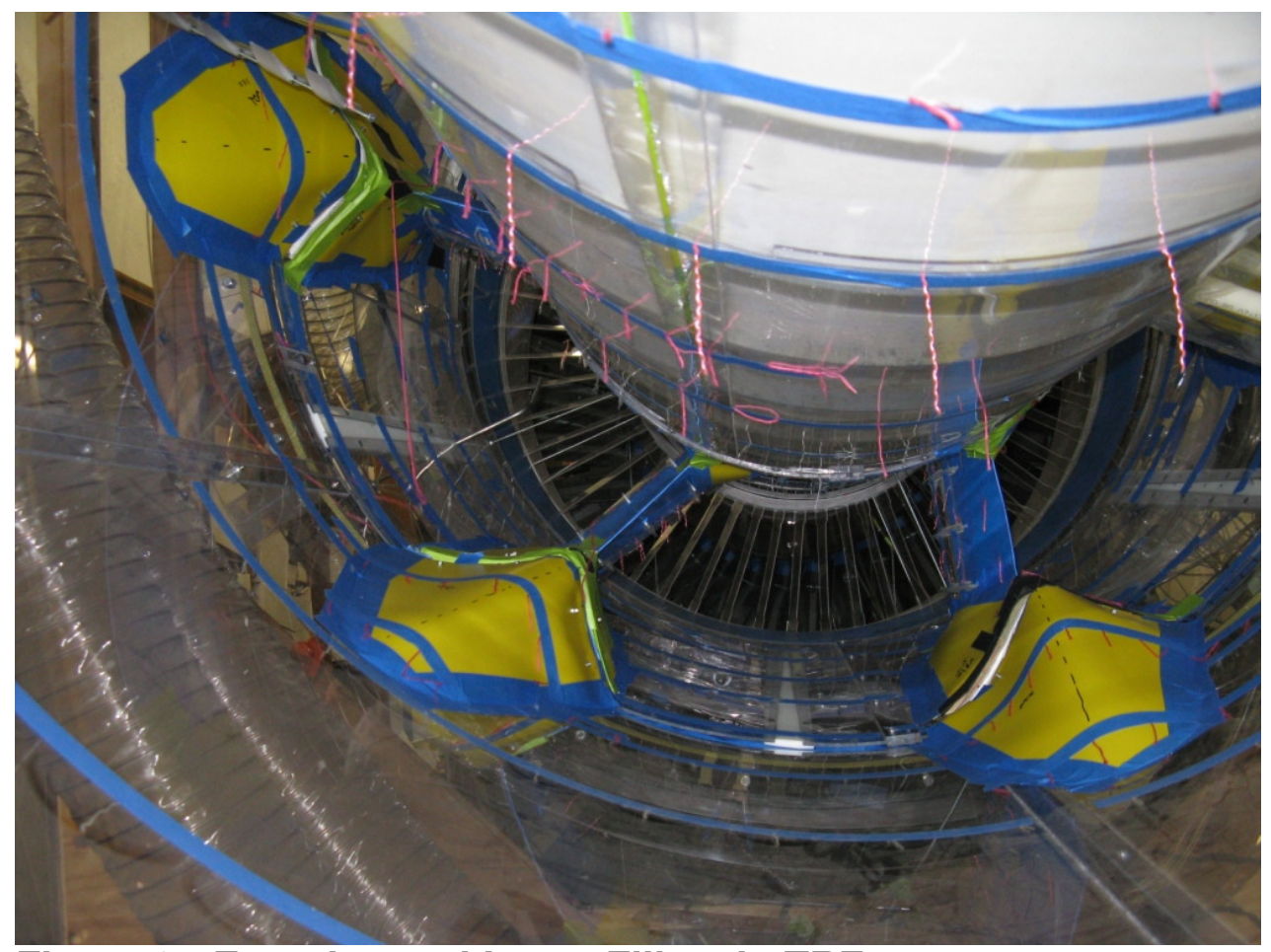

Figure 9: Experimental Large Fillets in TRF

The plane of the traverse cuts through the large fillets, which were cut at the traverse plane which allowed the traverse to be made. Outside of the fillet dead zones so created, the strut wake is greatly reduced. The large fillet cross section area was calculated to reduce the area ratio of the exhaust cylinder nearly to unity. That is, there was little potential for diffuser pressure recovery benefit at the TRF outlet, station 27. Regardless, these fillets were left in place to facilitate the strutlet test repeatability. Looking farther downstream, the annular flow area at the maximum thickness of the two large struts in diffuser Section 2 is only slightly larger than the Station 27 flow area with the Figure 9 fillets in place. Thus, losses associated with area changes were very minimal, but other losses associated with local flow direction changes were much increased by these very large fillets. The fillets were effective for the intended use, but do not seem especially promising as a practical turbine flow improvement because of the directional change losses. This is not to say that a small fillet could not have a net benefit.

Most turbines have streamlined sharp trailing edges on the exhaust struts. Therefore, fairings were added to each strut, which also reduced the stall size behind each strut. However, the $\mathrm{Cp}$ of the exhaust cylinder or the entire exhaust system was not necessarily improved. This lack of improvement was attributed to the low area ratio of the TRF with the big fillets, and with the entire diffuser attached, to the much lowered turbulence in downstream sections. Regardless, adding a fairing to the test strut seemed useful in maintaining test repeatability, and even flow stability within a test. The fairings were left in place to facilitate comparative strutlet tests. 


\section{$\underline{\text { Test Strutlets }}$}

Four strutlets were fastened to the hub and rim, near the strut leading edge. Initial positioning was done by hand. Then, the strutlet was fixed in position with 6-32 screws, (approximately 0.13 inches in diameter.) The screws were attached to either the strut or the diffuser walls in line with the strutlet.

Three of the test strutlets are shown in Figure 10.

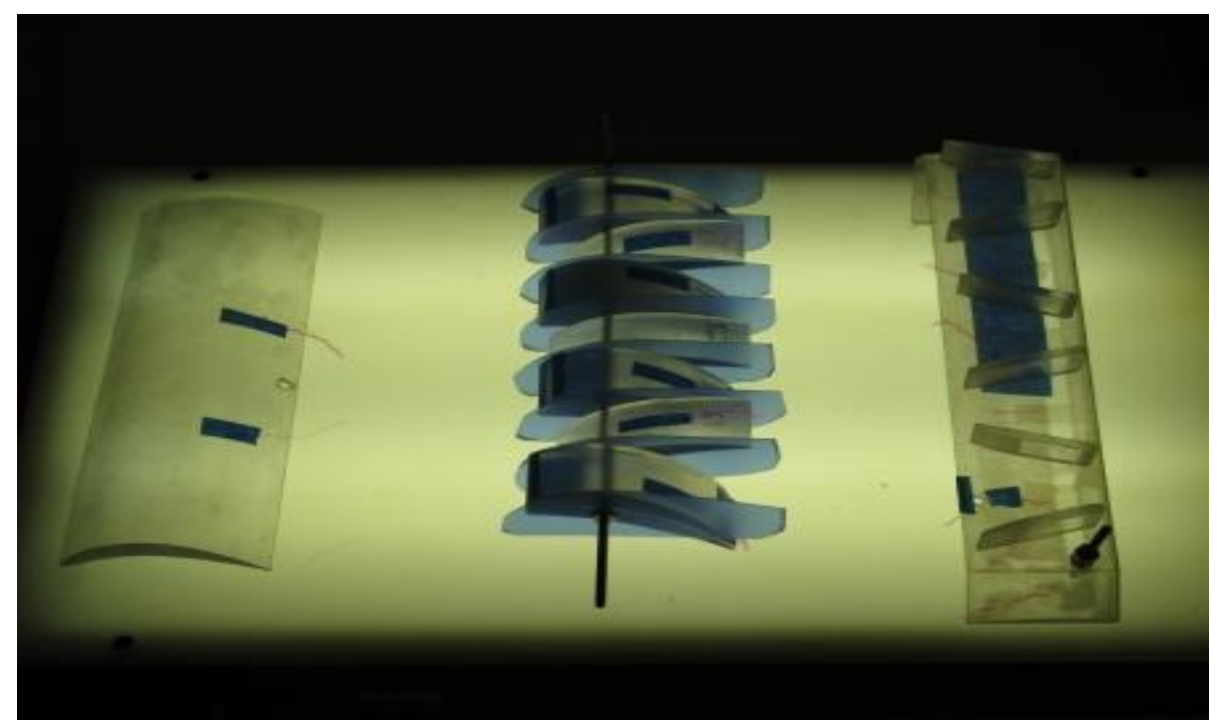

Figure 10. Strutlet Types and a Slat Tested

Figure 11 shows one strutlet in test position. Other strutlets were tested in approximately the same position, with minor adjustments for best performance. 


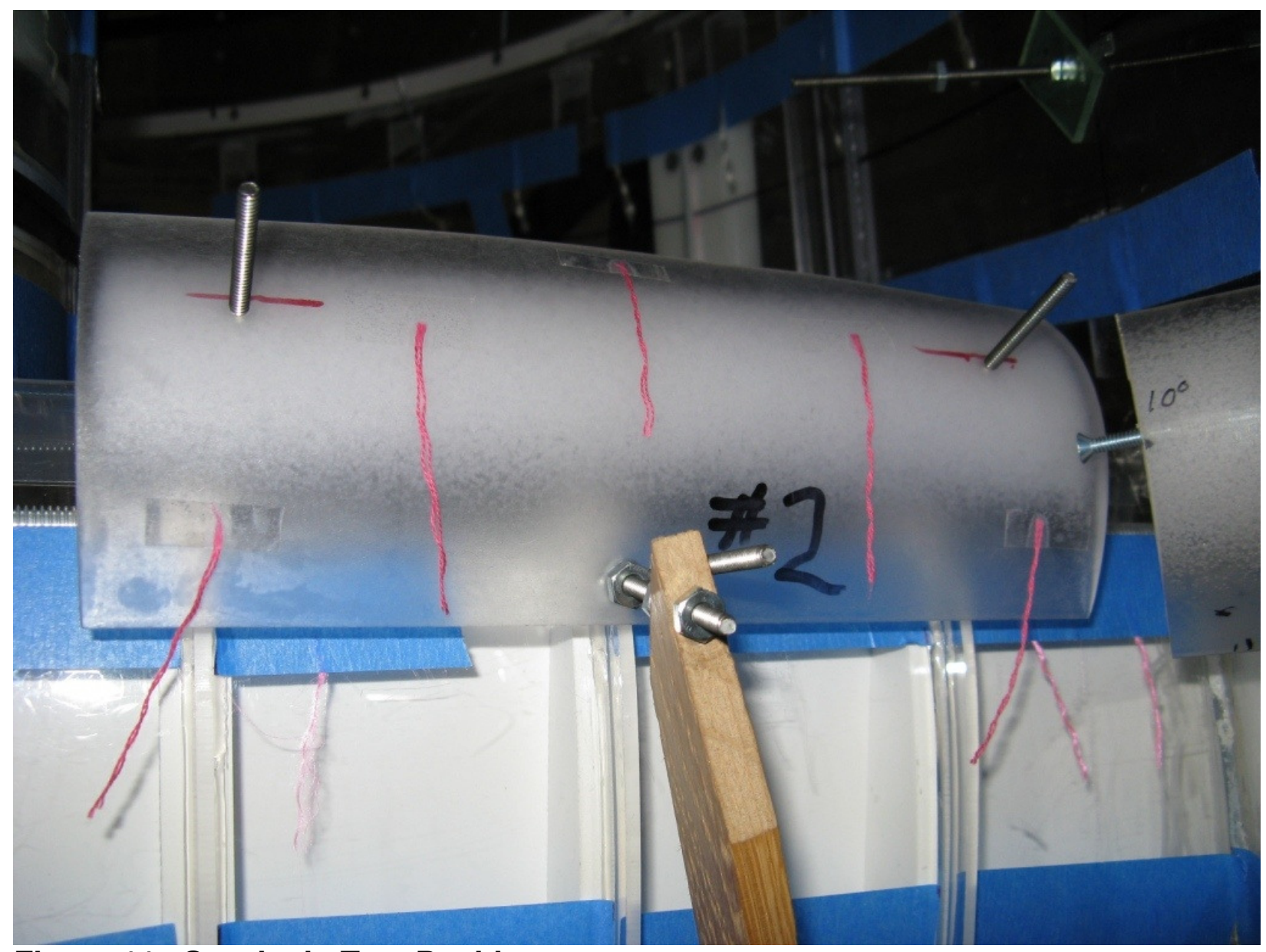

\section{Figure 11. Strutlet in Test Position}

Test Item 1, not shown, is of a "dragon tooth" strutlet with a 3-inch chord. The "teeth" are formed by simply cutting notches in an airfoil, removing the trailing third. The notches are cut 0.75 inches wide at 1.5 inch pitch. The possible benefit is that of both a slat and vortex generators. Drag is very high.

Test Item 2, seen at the left side of the figure, is a 3-inch-chord airfoil installed as a leading edge slat. The airfoil approximates a NACA- 65 series flow shape, cambered to match the strut leading edge. The laminar flow feature of this shape is not an advantage or disadvantage in this application; no-cost and immediate availability of an aerodynamic profile were the compelling attributes when choosing this airfoil.

Test Item 3, not shown, is a smaller slat, 2-inch chord, cambered to match the strut leading edges. There are no vortex generating features.

Test Item 4 is a strutlet similar to Test Item 3, but has vortex generators attached.

Test Item 5 is a short airfoil placed in the acute 60 degree corner between the strut and outer rim. It is a highly cambered, 3-inch chord laminar airfoil shape. It did reduce the corner stall and was in place for all tests excepting baseline. 
Test Item 6 is baseline and no strutlet, leaving just the TRF strut, with nothing added.

The center item in Figure 10 had exceptionally high drag.

Table 1 shows the test results. Results of three of the more significant tests are reviewed here.

Looking at the table, there are three total pressure traverses on the suction side of each strut for each test configuration, the first near the hub, the second mid-span on the strut, and the third the diffuser outer rim. The criterion for judging is the strength of flow near the surface (attachment), and lack of large low-flow zones elsewhere.

Looking at the hub traverse, the baseline shows a clear advantage over any of the strutlets. Near the strut surface, all strutlets improved flow slightly. However, at $2 \mathrm{~cm}$ and farther out from the strut, the baseline shows the highest velocity. Curiously, the corner vane, which is nowhere near, caused a $1.0 \mathrm{~cm}$ thickening of boundary layer, presumably because locally, the pressure gradient became more adverse and $\mathrm{Cp}$ was slightly improved locally. The two vortex-generating strutlets performed less well than the two simple slats, presumably because of their high drag.

Along a mid-strut traverse, the two vortex-generating strutlets and the large slat strengthened the boundary layer against the strut, but only slightly. The corner vane tested alone created a large full stall, and was rated far below any other test. 
Table 1: Total Pressure Traverses on Struts with and without Strutlet, Baseline Exhaust Cylinder Exhausting into Room (No Diffuser)

Strut No. Strutlet

Travrs.No. Type

\author{
distance $(\mathrm{cm})$
}

DragonTooth

Test 13 3" chord, 3/4 wide "teeth"

28-Feb at 1.5"pitch Pt (in.H2O)

Traverse 1 \&cornr.vn

Traverse 2

Traverse 3 $-0.09$

$\begin{array}{ll}-0.1 & -0.07\end{array}$

$-0.05$

0.16

$\begin{array}{llll}0.07 & 0.09 & 0.14 & 0.26\end{array}$

0.2

0.28

0.26

$\begin{array}{lllll}0.16 & 0.17 & 0.14 & 0.07 & 0.05\end{array}$

$0.04 \quad 0.03$

$0.01-0.02$

$\begin{array}{ll}-0.03 & 0.02\end{array}$

0.31

0.05

$0.08 \quad 0.07$

$0.06 \quad 0.05$

Test 2 Solid slat

28-Feb 3" chord

Traverse 1 \&cornr.vn.

Traverse 2

Traverse 3

$\begin{array}{rrrr}0 & -0.02 & 0.05 & 0.16 \\ 0.11 & 0.19 & 0.15 & 0.14 \\ 0.09 & 0.16 & 0.17 & 0.06\end{array}$

0.2

0.27

0.27

$\begin{array}{ll}0.29 & 0.28\end{array}$

$\begin{array}{rrrr}0.26 & 0.25 & 0.25 & 0.2 \\ 0.29 & 0.3 & 0.29 & 0.2 \\ & - & - & \\ -0.05 & 0.04 & 0.03 & -0.0 \\ & & & \\ & & & \\ & & & \\ 0.31 & 0.29 & 0.26 & 0.2 \\ 0.27 & 0.3 & 0.31 & 0 \\ 0.06 & 0.05 & 0 & -0.0 \\ & & & \end{array}$

0.26

0.27

0.29

0.06

$0.02 \quad-0.02$

$-0.05$

$-0.05$

$\begin{array}{rrrr}0.26 & 0.25 & 0.25 & 0.2 \\ 0.29 & 0.3 & 0.29 & 0.2 \\ & - & - & \\ -0.05 & 0.04 & 0.03 & -0.0 \\ & & & \\ & & & \\ & & & \\ 0.31 & 0.29 & 0.26 & 0.2 \\ 0.27 & 0.3 & 0.31 & 0 \\ 0.06 & 0.05 & 0 & -0.0 \\ & & & \end{array}$

$\begin{array}{rrrr}.26 & 0.25 & 0.25 & 0.2 \\ .29 & 0.3 & 0.29 & 0.2 \\ & - & - & \\ .05 & 0.04 & 0.03 & -0.0 \\ & & & \\ & & & \\ & & & \\ .31 & 0.29 & 0.26 & 0.2 \\ .27 & 0.3 & 0.31 & 0 \\ .06 & 0.05 & 0 & -0.0 \\ & & & \end{array}$

Test 3 Small slat

28-Feb 2" chord

Traverse 1 \&cornr.vn.

Traverse 2

Traverse 3

$\begin{array}{rrrrrrrrrrrr}-0.02 & 0.01 & -0.01 & 0 & 0.1 & 0.27 & 0.24 & 0.3 & 0.3 & 0.31 & 0.29 & 0.26 \\ -0.01 & -0.02 & -0.02 & 0.08 & 0.27 & 0.2 & 0.17 & 0.2 & 0.25 & 0.27 & 0.3 & 0.31 \\ -0.1 & -0.11 & -0.12 & -0.13 & -0.03 & -0.02 & -0.08 & -0.02 & 0.05 & 0.06 & 0.05 & 0\end{array}$

Test 6 None, 28-Feb Baseline

Traverse 1

Traverse 2

Traverse 3

$\begin{array}{rrrrrrrrrrrrr}-0.11 & -0.06 & 0.02 & 0.12 & 0.2 & 0.3 & 0.3 & 0.31 & 0.31 & 0.32 & 0.31 & 0.29 & 0.2 \\ -0.01 & 0.05 & 0.05 & 0 & 0.02 & 0.21 & 0.3 & 0.3 & 0.28 & 0.3 & 0.31 & 0.3 & 0 \\ -0.14 & -0.14 & -0.15 & -0.15 & -0.15 & -0.15 & -0.15 & -0.15 & -0.12 & -0.09 & 0 & 0.05 & 0.1\end{array}$

Test 4 2" Slat 28-Feb w/ VG's

Traverse 1 \&cornr.vn.

Traverse 2

Traverse 3

$\begin{array}{ccc}-0.05 & -0.01 & 0.04\end{array}$

$\begin{array}{ll}0.1 & 0.14\end{array}$

0.18

0.1

$\begin{array}{ll}0.24 & 0.2\end{array}$

$\begin{array}{ll}0.19 & 0.23\end{array}$

0.24

$\begin{array}{lll}0.02 & 0.1 & 0.1\end{array}$

$0.11 \quad 0.11$

0.16

0.15

$0.18 \quad 0.25$

$\begin{array}{ll}0.3 & 0.3\end{array}$

$\begin{array}{lllll}0.08 & 0.06 & 0.06 & 0.08 & 0.05\end{array}$

$0 \quad-0.02$

$\begin{array}{ll}-0.03 & -0.03\end{array}$

$\begin{array}{ll}-0.05 & 0.05\end{array}$

0.06

Test 5 Corner

28-Feb airfoil only

Traverse 1 no strutlet

Traverse 2

Traverse 3

$\begin{array}{rrrrr}-0.07 & 0.02 & 0.05 & 0 & -0.01 \\ -0.15 & -0.15 & -0.15 & -0.15 & -0.15 \\ -0.15 & -0.15 & -0.15 & -0.15 & -0.15\end{array}$

0.15

0.3

0.3

0.31

$\begin{array}{lll}0.3 & 0.31 & 0.31\end{array}$

$-0.15$

$-0.15$

$-0.15$

$-0.1$

$\begin{array}{ll}-0.02 & 0.08\end{array}$

$-0.15$

$-0.15$

$-0.15$

$-0.1$

0.05

$0.15 \quad 0.25$

Test 7 Baseline,

29-Feb w/fairing

Traverse 1 added to strut

Traverse 2

adrut

$\begin{array}{rrrrr}0 & 0.01 & 0 & 0.01 & 0.02 \\ 0.22 & 0.06 & 0.02 & 0.03 & 0.04 \\ 0.08 & 0.04 & -0.04 & -0.07 & -0.1\end{array}$

$0.12 \quad 0.24$

$0.3 \quad 0.31$

Traverse 3

0.08

0.04

$-0.1$

$-0.1$

$-0.1$

$-0.1$

$-0.1$

$\begin{array}{llll}0.31 & 0.31 & 0.31 & 0.3 \\ 0.14 & 0.18 & 0.2 & 0.8\end{array}$ 


\begin{tabular}{|c|c|c|c|c|c|c|c|c|c|c|c|c|c|}
\hline $\begin{array}{l}\text { Test } 8 \\
29-F e b\end{array}$ & $\begin{array}{l}\text { Baseline } \\
\text { w/fairing }\end{array}$ & & & & & & & & & & & & \\
\hline Traverse 1 & and corner & 0.04 & 0 & 0 & 0 & 0.02 & 0.08 & 0.18 & 0.29 & 0.31 & 0.31 & 0.31 & 0.32 \\
\hline Traverse 2 & airfoil & 0.14 & 0.09 & 0.03 & 0.01 & 0.01 & 0.05 & 0.06 & 0.09 & 0.08 & 0.1 & 0.09 & 0.14 \\
\hline Traverse 3 & & 0.11 & 0.06 & 0.04 & -0.05 & -0.02 & -0.15 & -0.15 & -0.14 & -0.13 & -0.1 & 0.11 & 0.09 \\
\hline $\begin{array}{l}\text { Test } 9 \\
\quad 29 \text {-Feb }\end{array}$ & $\begin{array}{l}\text { Above, } \\
\text { w/ strutlet } 1\end{array}$ & & & & & & & & & & & & \\
\hline Traverse 1 & dragon tooth & 0.05 & 0.01 & 0.02 & 0.04 & 0.06 & 0.1 & 0.14 & 0.15 & 0.15 & 0.14 & 0.12 & 0.15 \\
\hline Traverse 2 & & 0.21 & 0.15 & 0.06 & 0.05 & 0.06 & 0.11 & 0.13 & 0.12 & 0.1 & 0.1 & 0.09 & 0.11 \\
\hline Traverse 3 & & 0.2 & 0.15 & 0.08 & 0.04 & 0 & -0.02 & -0.05 & -0.07 & -0.07 & -0.07 & 0.08 & 0.06 \\
\hline
\end{tabular}

A "Zero" distance from the strut surface is actually a 0.25 copper tube total pressure probe pressed against 1 the surface.

2 Total pressure values less than -.15 are shown as -.15

Readings made using a Dwyer Magnehelic gauge, 0 to 0.50 in. W. C.

3 range.

Considering the traverse nearest the diffuser rim, the best result in the boundary layer was the large slat, followed by the two vortex generating strutlets. The smaller slat was poor. Both the corner vane only and baseline were in full stall.

Overall, the large plain slat performed best, while others have their particular advantages, primarily a better-mixed boundary layer. All were effective to a degree at reducing boundary layer thickness on the strut suction side. However, farther out into the free stream, the deep strutlet wakes offset this advantage.

The low energy flow at a distance out from the strut is caused by strutlet drag. A strut with efficient flow would perform with only a small wake. However, wakes observed were large, indicating high drag.

The following section reviews an item not envisioned when proposing this project, which are the large corner stalls. None of the strutlets affected the large corner stall at each strut-rim intersection. These stalls must be successfully eliminated in flow leaving this diffuser section prior to effective use in a smooth-flowing, low noise and vibration, high area ratio diffuser. It was not known in Phase I if the stalls were strictly an artifact of the model flow, or instead, common in real turbines as well. Spot measurements of two large turbines (not a part of this project) showed modest corner low-flow zones downstream of the TRF. In one case, this appeared to be not significant, or of modest impact. In the other, the corner stall was the leading candidate for the source of a lowflow zone that greatly enlarged downstream, with lower than expected diffuser performance, with low frequency noise and duct vibration. 


\section{A Boundary Layer Issue}

The boundary layer and corner stall issue dominates the results, so merits further discussion. The persistent low flow or corner stall zones on the rim and hub intersections with the struts, with or without fairings or with small fillets, must have a source. One candidate is the entering boundary layer.

To test this hypothesis, the diffuser was axially separated 0.25 inches from the inlet duct immediately upstream, a manifold provided around the periphery, and a 20 horsepower suction fan (New York Blower Size 19 with DH centrifugal wheel) was connected. This system could easily draw off the adjacent half inch of the boundary layer, leaving much less boundary layer. Figure 12 shows total pressure traverses out from the rim and hub surfaces along two lines, to show boundary layer thicknesses. (This is the test setup used for all strutlet tests.) The angular convention is, looking upstream, 0 degrees is straight up, 120 degrees is at "four o'clock", and 180 degrees is straight down. The two dashed lines are at Station 6, or six inches into the TRF diffuser. The start of boundary layer growth is just becoming visible on the lowest dashed line.

\section{Total Pressure in Boundary Layer at Diffuser Inlet and 6 Inches into Diffuser}

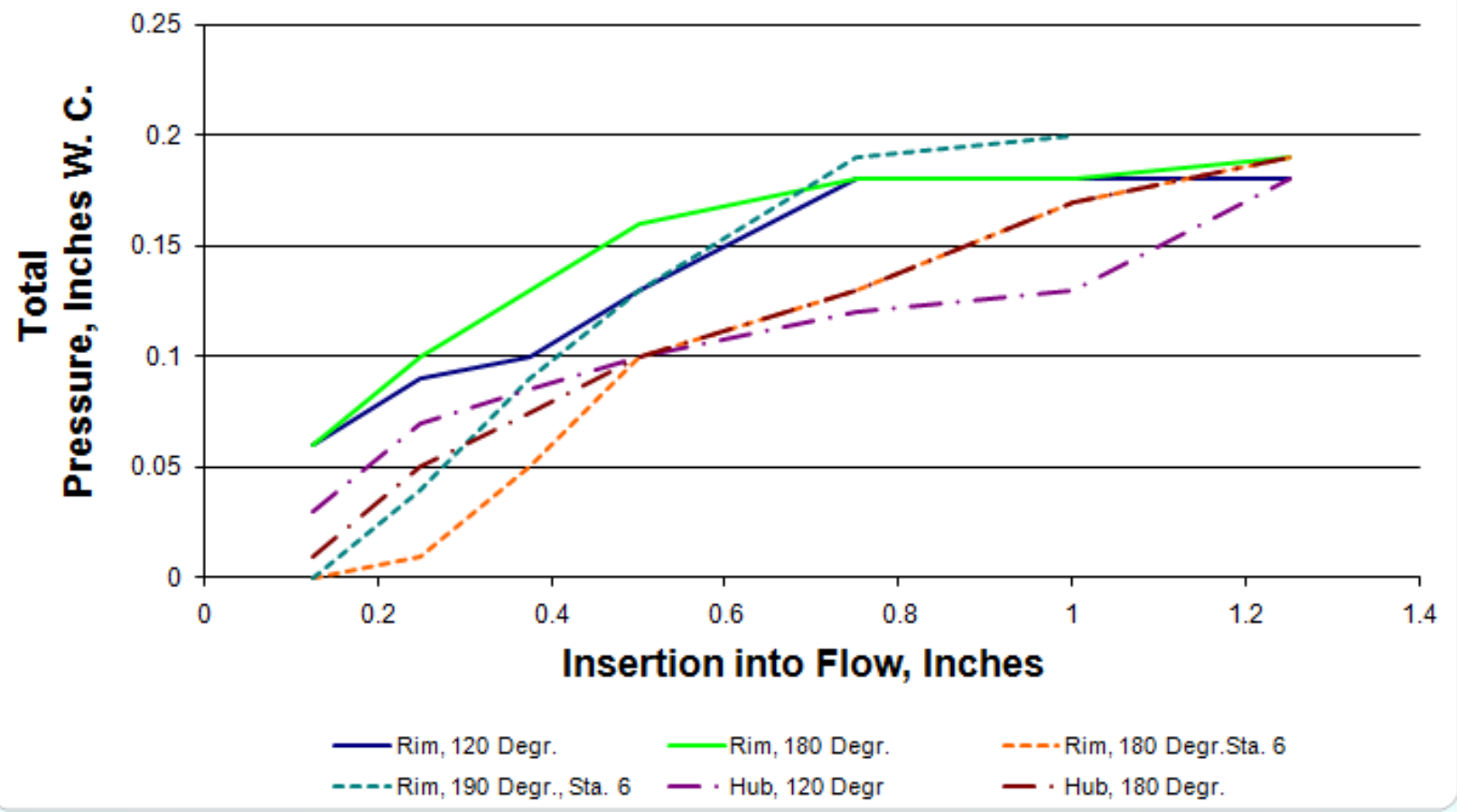

Figure 12. Total Pressure in Boundary Layer at Diffuser Inlet 6

At 22 degrees swirl, with the suction fan "on," the corner stalls remained, though a noticeable size reduction occurred. 
Next, small fillets were added to the struts at the intersections with the hub and rim. Perhaps the stalls were reduced a bit, but not greatly.

The conclusion is that, without any other apparent source of slowed gas, these stalls or slow zones must be mostly boundary layer gas generated within the diffuser, then drawn along the walls toward and concentrated at a location on or near the suction side of each strut. The stalls, as were shown in Figure 8, were judged large enough that a downstream diffuser would not perform optimally. A quick connect with the entire diffuser system, area ratio 6.18 , showed this to be true.

Phase I work ended at this point, although CIEA pursued independently sponsored research afterward.

\section{CONCLUSIONS OF PHASE I TESTS}

1. The strutlet concept as tested has too much drag to be of practical use.

2. The strutlet concept was effective at reducing stalls on the struts, at 20 degrees swirl and a strong adverse pressure gradient.

3. The strutlets did not reduce the corner stalls at the strut-rim and strut-hub intersections.

4. Any practical strutlet would require that drag be greatly reduced.

5. The strut intersection or corner stalls observed are large enough to be significant if they occur in real turbines.

\section{ACKNOWLEDGEMENTS}

I am very appreciative of the efforts of Mr. Hall, Ph.D., and Mr. DeAnda for their tireless efforts while conducting tests and in data creating the data contour plots, and for their enthused encouragement. I would also thank the diffuser and industry consultants, for offering their vast experience and willingness to share. The work would not have been possible without the support of the Department of Energy for the Phase I SBIR project, which is greatly apreciated.

\section{Reference}

Spangler, Jack G., and Sinclair Wells, Jr.: "Effects of Spiral Longitudinal Vortices on Turbulent Boundary Layer Skin Friction", NASA Contractor Rport No. CR-145. 1964. 


\section{APPENDIX A}

Appendix A presents highlights of independently sponsored work extending beyond Phase I.

This work was planned as a "competition" set up between two design concepts. The first concept uses augmented turbulence to improve diffuser performance. The second concept uses strutlets to suppress turbulence and, in the role as vortex generators, possibly boost performance. First, however, the model measurement capabilities required reduced uncertainties, and the corner stalls noted in Phase 1 needed to be eliminated. A simple low frequency pressure fluctuation measurement was added near the end of the work, as an indicator of potential to generate low frequency noise at the exhaust stack outlet. Such low frequency noise is, at some turbine sites, of substantial concern.

\section{Improving the Test Setup}

First, the inlet flow path upstream of the diffuser was redesigned. The inlet section was lengthened, "bleed off" slots installed to remove more of the inner and outer boundary layers, the second swirl vane set was removed, and the larger-scale flow turbulence modestly reduced with a set of screens at the fan. The result is that flow entering the diffuser could have almost any desired "blockage" (boundary layer displacement thickness total area divided by total inlet area) at the hub and rim. Also, the boundary layer profile could be adjusted, including a profile with slightly more velocity pressure in the inner and outer 0.75 inch, to help counter boundary layer growth between the screens and diffuser inlet. This setup gave less control of the swirl adjustment, which was judged acceptable. The swirl was reduced from Phase I values of 20 degrees hub, 10 degrees rim to an average of 11 degrees. A survey showed a range of 8 to 14 degrees, depending mostly on circumferential location. The hub boundary layer bleed locations and gaps were at stations 0 (1/16 inch gap), station 24 (3/4 inch gap) and just upstream of the screen at the fan (2.5 inch gap.) The rim boundary layer (BL) bleed gaps were at the same stations and approximately the same gaps.

Rim exhaust bleed is to two outer annuli, maintained at reduced pressure by a suction fan. The Hub bleed slots exhaust into the central hub, ducted to the suction fan through the center body and then struts. The rim and hub upstream bleed slots remove a layer of flow roughly two inches thick, and the next slot at the measurement section inlet removes approximately one-half inch of flow. These first two slot pairs do not affect measurement accuracy, being adequately upstream of the static probes. The small slot at station 0 has a suction equal to the local static pressure plus about 20 to 50 percent of local velocity pressure. This slot thus removes a layer estimated at up to $1 / 32$ of an inch.

\section{Discussion of Some Error Sources}

A potential error source is the bleeds at station 0 , as these are downstream of the static pressure holes. Their purpose is to prevent small local stalls at the 8 degree direction change at the TRF, which if present, would create an uncertainty. These bleed slots are 
adjusted by small screws set to $1 / 16(0.0625)$ inch. The slot suction differential suction was set to about half or less of the calculated velocity pressure at station 0 . The estimated bleed flow is certainly less than 1 percent of total flow, and we estimated up to 0.5 percent. The estimated error is to increase the $\mathrm{Cp}$ by up to 0.02 , and by our estimate, 0.01 or less. Indeed, no noticeable data shift was noted when these slots were temporarily covered with adhesive tape.

A second error source is that the total pressure is measured at only eight locations, all at the same insertion of about 4 inches (25 percent insertion) into the flow. For the described tests of just the TRF, the total pressure was set constant around and across the annulus, and the errors are modest, provided that the flow pattern is nearly constant between tests. The latter requires that the flow profile be checked if there are any major changes in diffuser $\mathrm{Cp}$ performance, such as adding a section of diffuser, because of flow changes through the fan outlet guide vanes. Slightly uneven flow from the screens also contributes an uncertainty. Significantly, when testing at $\mathrm{Cp}$ values over about 0.7 , which always occurred when testing with the entire diffuser connected, the fan outlet flow radial Pt profile became stronger near the rim than near the hub. So, there is a slight systematic error, most likely leading to lower measured $\mathrm{Cp}$ values that would have been measured with the intended uniform inlet flow. That is, the Pt locations caused a probably underreported $\mathrm{Cp}$. This error is estimated at +0.01 to $-0.02 \mathrm{Cp}$ units.

Another error source when testing a long diffuser is the condition of the joints between diffuser sections. This error was noticed when attempting to repeat a test after diffuser disassembly and reassembly. If the measured difference was over about $0.005 \mathrm{Cp}$ units; a diffuser inspection should follow, as an assembly problem would inevitably be found. With good reassembly, the repeatability was better than 0.010 , and usually within $0.005 \mathrm{Cp}$ units. This maximum random error with correct assembly is estimated at up to $+-0.008 \mathrm{Cp}$ units.

Overall, data repeatability was found acceptable, with agreement between repeat tests after correct reassembly, days or weeks apart, at $+-0.01 \mathrm{Cp}$ units, and typically half that.

\section{Diffuser Pressure Measurements}

Diffuser static pressure was measured in two arrays of wall taps, one circumferential and the other axial. All wall taps were simple 1/8 inch holes. The axial profile tap array consisted of a line at about 9 o'clock, looking upstream. There are 34 static holes, more closely spaced near the diffuser inlet than downstream. The second type of static measurements is circumferential averages, measured at four stations. Each ring consists of eight taps, equally spaced around the circumference. Pressure averaging for each ring is at a small manifold connected to all eight holes. The discussed tests are all analog averaged. All static taps are connected to the Scanivalve transducer, using PVC plastic tubing with an inside diameter of nominally $1 / 8(0.125)$ inch. The tubing lengths vary, which is a source of phase error and, above a higher frequency, of amplitude error. These errors are believed significant above approximately $5 \mathrm{~Hz}$, and possibly at 
lower frequencies, too. These phase and amplitude errors do not affect the $0 \mathrm{~Hz}$ component of the pressure, which is used in the Cp calculations.

The usual test run consisted of a record of 240 data point sets for each static tap or ring, each data point representing an average of 5 instantaneous measurements. The 240 data points were recorded at 250 millisecond intervals, giving a Nyquist frequency of 2 $\mathrm{Hz}$.

For selected tests, the averages were done 25 times per second, again with five samples per average. Thus, the Nyquist cutoff frequency is $12.5 \mathrm{~Hz}$, with a measure of safety against aliasing due to the averaging of instantaneous samples being taken at $125 \mathrm{~Hz}$. These $25 \mathrm{~Hz}$ data have not yet been analyzed.

Low frequency pressure variations were noticed and readily measureable, and could be used for a comparison between tests. However, above about $5 \mathrm{~Hz}$, accuracy is compromised as previously noted, and the data are not suited for uses other than comparisons between tests on this diffuser model. Should the low frequency fluctuation or noise data become important, selected tests could be re-run with the tubing lengths equalized and shortened, and possibly the tubing diameter increased.

A frequency correlation of turbulence-related low frequency noise between model and actual is of limited but important use. There are Reynolds number effects, and potentially inlet turbulence effects.

The large eddy passage frequency, corresponding to a potential noise generator, depends on the velocity and size scale factors. The model data presented were recorded at a diffuser inlet velocity of near 50 feet per second, while the full scale turbine was at near 1000 feet per second. The model to turbine physical size scale factor is 0.376 to 1 . Thus, $10 \mathrm{~Hz}$ on the model is, on the actual turbine 10 $\mathrm{Hz}^{*}(1000 / 50)^{*} 0.376=75.2 \mathrm{~Hz}$. So, the model data at $0-2 \mathrm{~Hz}$ would have some correlation with turbine frequencies of $0-15 \mathrm{~Hz}$. Note that there are also potentially Mach number effects, presumed to be quite modest in magnitude, and not included in this simple scaling calculation.

Pressure scales with the velocity pressure of the flow, presuming low-speed subsonic flow. Thus, a 0.5 inch w.c.model velocity pressure corresponds to 80 inches on the actual turbine. Extending this ratio to acoustic pressures is theoretically possible, but given the many sources of uncertainty, it is suggested that the fluctuation data presented should be used only for rank ordering of model configurations. The main flow fan and the boundary layer suction fans are both interfering low frequency noise sources.

In particular, we found that the acoustic pressure in the model diffuser inlet at $2 \mathrm{~Hz}$ and below (15 Hz actual turbine) was quite sensitive to how we modeled the diffuser inlet section (TRF) flow loss items. Also, we did not model cooling flows entering at the inlet rim and hub, or a series of smaller ledges downstream. Instead, for best data 
repeatability, we instead pulled a tiny amount of air out of the diffuser inlet where the actual turbine has a modest entering flow. Perhaps most significantly, we never did the work needed to drop the $\mathrm{Cp}$ down quite to the 0.68 to 0.72 typically measured on the actual turbine.

Corner stalls were reduced mostly by limiting maximum swirl to 14 degrees, compared to formerly 10 at the rim and 22 at the hub. We also changed turbulence characteristics in the incoming flow, which lessened the cross-flow component in the local boundary layer. In particular, reducing larger scale turbulence, and increasing smaller scale turbulence seemed to help "fix" the severe corner stalls. Also, we noted that the nose of each strut had a slightly smaller radius than intended, which was also fixed. However, increasing the nose radius alone did not seem to have a great effect. Thus, the corner stall phenomenon is at least partly, and perhaps mostly, a boundary layer secondary (or cross) flow issue. It is also an artifact of the scale model flow turbulence parameters. However, several full scale turbine data points (not a part of this project) are suggestive of lower than expected flow near the corners, too. Regardless, the corner stall phenomenon in turbines must be much less important than originally seen in the model.

Strutlet drag has been reduced. This was done after Phase I, and is still in progress.

\section{Tests Run}

After Phase I, tests were run to investigate, understand and eliminate the corner stall phenomena, to reduce the drag of the strutlets, and how strutlets might be applied in the TRF and downstream in a practical manner. Selected data are presented.

Figure A1 shows a typical Cp vs axial station.

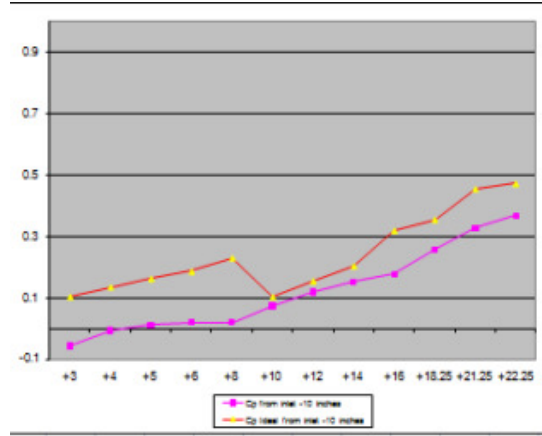

inr

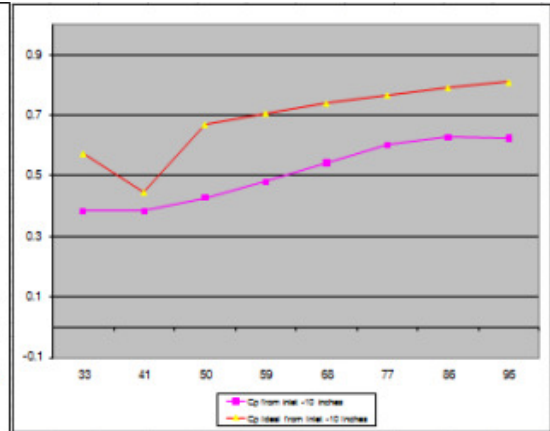

st

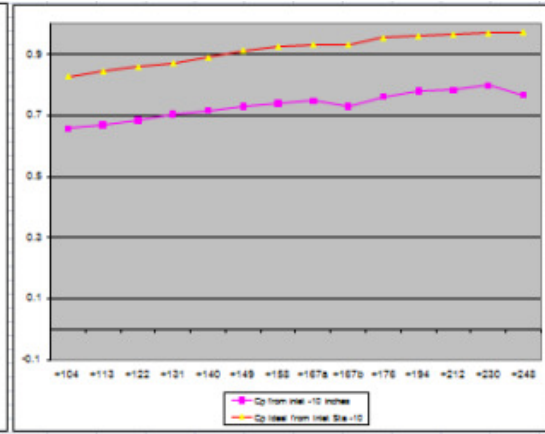

$\infty 2$

Figure A1: Ideal and Measured Cp from Inlet vs Station in Inches from Inlet in Mid-Performance High-Turbulence Model Diffuser Note Dips in Cp Ideal at Stations 10 through 12.25, and 33 through 50, Due to Strut Thickness. Step Expansion is at Station 167.

On Figure A1, the inlet is at the left, and the outlet on the right. This is a typical mediumperformance, high turbulence diffuser setup. This diffuser depends on turbulence of the proper (undetermined) scale for efficiency, and was found to be is very forgiving of any local low-energy flow zones in the inlet flow or downstream of struts. On the graph, the axial station scale is compressed at the large end of the diffuser. The ideal $\mathrm{Cp}$, denoted $\mathrm{Cpi}$, at the diffuser outlet, is 0.974 , with the $A R=6.18$. The actual overall $\mathrm{Cp}$ is at 0.801 , 
and the efficiency is then $0.801 / .974=0.822$. Other than presumed duct vibration and low frequency noise, this is a practical diffuser. At the far left of Figure A1, the apparent $\mathrm{Cp}$ drops below 0 . Because of a local static pressure depression at the inlet, flow direction changed from cylindrical to expanding into an 8 degree cone. The calculated Cpi, being based only on area of the flow passage, clearly shows two local depressions associated with the struts. The first strut set has leading edge at station 8. Surprisingly, the measured $\mathrm{Cp}$ does not show these dips, being measured between struts. In fact, the $\mathrm{Cp}$, if measured in the central half sector of the flow passages between struts, shows almost no diffuser effect (rise in static pressure) from stations 0 to 8 , staying below $\mathrm{Cp}=0.02$. (This has a significant implication, suggesting that the baseline actual turbine inlet static pressure measurement in Phase I, taken at 10 inches from the turbine wheel and not close to a strut, is valid with no significant correction for diffuser performance upstream of the measurement station.)

In the Phase I baseline model tests, at the TRF (turbine rear frame) outlet, Station 27, locally the $\mathrm{Cpi}=0.53$, but the measured $\mathrm{Cp}$ is only 0.37 , for a rather poor efficiency of $0.37 / 0.53=0.70$. Thus, the flow has not "stuck" tightly to one or more surfaces. Most of this "missing" energy is in the free stream flow away from the walls and struts, and has not yet been dissipated to heat by turbulence. It is thus still available for productive use, farther downstream. The center panel of Figure A1 shows about the same gap between the Cpi and $\mathrm{Cp}$ from left to right, indicating continued rapid mixing of the fast, unrecovered free stream flow exiting the TRF. The third or right panel shows that the gap between the $\mathrm{Cpi}$ and $\mathrm{Cp}$ is still nearly constant, indicative of continued efficient mixing by turbulence. The efficiency has risen from 0.70 at the TRF outlet to about 0.80 at the final diffuser outlet. Strong large-scale downstream turbulence has been of great benefit for this diffuser's efficiency.

In the follow-up work, the TRF flow performance increased to typically $\mathrm{Cp}=0.47$, for an efficiency of $0.47 / 0.53=0.89$. I categorize this as acceptable, considering this TRF has features that each contribute a small inefficiency, even if each feature represented good industry practice when originally designed.

Table A1 shows data from three significant tests of the baseline diffuser shell. 


\begin{tabular}{|c|c|c|c|c|c|c|c|}
\hline Configuration & $\begin{array}{l}\text { Character } \\
\text { of Flow }\end{array}$ & $\begin{array}{l}\text { Outlet } \\
\text { Flow }\end{array}$ & $\begin{array}{l}\text { Cp } \\
\text { Average }\end{array}$ & $\begin{array}{l}\text { Cp } \\
\text { Variation } \\
\text { RMS }\end{array}$ & $\begin{array}{l}\text { Cp } \\
\text { Extreme } \\
\text { Range of } \\
\text { Fluctuation }\end{array}$ & $\begin{array}{l}\text { Est. Press } \\
\text { (if } P v=80 \text { in. } \\
\text { (in. w.c.) } \\
\text { RMS }\end{array}$ & $\begin{array}{l}\text { wre variation } \\
\text { w.c.at inlet) } \\
\text { dB re } \\
20 \mathrm{microPa}\end{array}$ \\
\hline Baseline & Very Rough & $\begin{array}{l}\text { Voids and } \\
\text { Hot Spots }\end{array}$ & 0.758 & 0.014 & 0.071 & 1.11 & $143.9 \mathrm{~dB}$ \\
\hline $\begin{array}{l}\text { Augmented Turbulence } \\
\text { (Test with highest } \mathrm{p} \text { ) }\end{array}$ & Rough & $\begin{array}{l}\text { Voids and } \\
\text { Hot Spots }\end{array}$ & 0.824 & 0.008 & 0.042 & 0.62 & $138.8 \mathrm{~dB}$ \\
\hline $\begin{array}{l}\text { Supressed Turbulence } \\
\text { (Strutlett test with } \\
\text { highest } \mathrm{Cp} \text { ) }\end{array}$ & $\begin{array}{l}\text { Much Less } \\
\text { Rough }\end{array}$ & $\begin{array}{l}\text { Entire Area } \\
\text { Filled, but } \\
\text { not evenly }\end{array}$ & 0.817 & 0.005 & 0.026 & 0.38 & $134.6 \mathrm{~dB}$ \\
\hline
\end{tabular}

Notes:

1. Data are pressures averaged over eight wall static holes at 10 inches upstream of diffuser inlet, and eight total pressure tubes 4 inches from wall at 50 inches upstream of diffuser inlet.

2. Sampling is with Scanivalve unit set to sample at $20 \mathrm{~Hz}$, then report the average after every 5 th sample, 240 reports total. Thus, the reporting frequency is at $4 \mathrm{~Hz}$. The Nyquist frequency cutoff is $2 \mathrm{~Hz}$, Model.

3. Frequency scales by roughly a 7.5 factor: $2 \mathrm{~Hz}$ on model is about $15 \mathrm{~Hz}$ on actual turbine, presuming 80 inches velocity pressure at diffuser inlet.

4. Pressure data transmission tubes are apporoximately $10 \mathrm{ft}$. long, and 1/16 inch ID. Lengths are not equal.

5. Data are suitable for rank ordering model diffuser probable acoustic output. Other uses should be with caution.

Specifically, there are many sources of ucertainty in any exact linking of model and turbine sound levels.

Table A1 compares performance between diffusers with interior features that result in three different flow patterns. The time-averaged $\mathrm{Cp}$, RMS fluctuations in the $\mathrm{Cp}$, and the extreme range of $\mathrm{Cp}$ during each test are shown. The first configuration is a near baseline, the second has augmented turbulence, and the third has suppressed turbulence.

In the first configuration, the baseline flow was rough to the point of visibly vibrating the model's surface, causing the model to swing from its suspension, and causing noticeable low-frequency noise above that normally noticed from the model's fans. The diffuser outlet flow included hot spots and local transient reversals. Later, it was noticed that this particular test seemed to have more pulsing than most of the similar configurations tested. The actual turbine diffuser, too, showed intermittent episodes of pulsing flow. At roughly 3 feet of probe insertion, just upstream of a large step expansion, at station 37, flow sometimes repeatedly dropped to zero for a second or several seconds, and would at times "puff."

The second configuration is optimized for high downstream turbulence. This may be termed an augmented turbulence diffuser, in that the good $\mathrm{Cp}$ performance is dependent on rapid mixing due to strong turbulence transverse to flow. Despite the 
extra turbulence, we noticed less overall model vibration than in the baseline test. Indeed, the RMS variation of the $\mathrm{Cp}$ is less, at about 60 percent of that in the first model test.

The third test is of a diffuser with suppressed turbulence. The shell, center body, and six TRF struts are unchanged from above. However, the two strut sets in the main diffuser are completely reconfigured, there are added strutlets, and other additions judged suitable for a smooth-flow retrofit kit. Compared to the previously discussed augmented turbulence test, the $\mathrm{Cp}$ is lower by 0.007 , which for an 80 inch inlet velocity pressure, would be 0.6 inches more backpressure on the turbine. However, the $\mathrm{Cp}$ is quite steady, with the lowest fluctuation of any test checked, corresponding to 0.4 inches RMS on the actual turbine. If the RMS variation is taken to correspond to a scaled low frequency noise generation mechanism, then compared to the baseline, the two higherperforming diffusers discussed generate 5 and $9 \mathrm{~dB}$ less low-frequency acoustic energy, respectively.

The conclusion is that in the stable but highly turbulent flow case, and in the suppressed turbulence case, the low-frequency pressure variation was reduced significantly. However, the amounts of reduction shown should not be taken as anywhere near exact. That would require a sophisticated, multi-channel acoustic analysis on the model and a real turbine.

The backpressure reduction that would be achieved by the diffuser using strutlets is calculated for two cases: On Table A1, the model baseline $\mathrm{Cp}=0.758$, and the strutlet system is at $\mathrm{Cp}=0.817$, for an improvement of $0.059 \mathrm{Cp}$ units. Alternately, using actual turbine baseline Cp data, and taking the baseline Cp as 0.72, the improvement is 0.097 Cp units. In terms of actual turbine backpressure, presuming 80 inches velocity pressure at the diffuser inlet, the improvement covers a rather wide range, from 4.7 to 7.8 inches. The two numbers vary depending on which baseline $\mathrm{Cp}$ is used-actual turbine or scale model. (The model "baseline" performance is better than the actual turbine baseline.) Using these two baseline values, in terms of turbine power and heat rate, at a conversion of 9 inches exhaust pressure reduction per percent power and heat rate improvement, the range is 0.52 and 0.87 percent, simple cycle. This range is above the project proposal stated goal of improving turbine power and heat rate by 0.5 percent. However, in a combined cycle plant, typical for the Siemens-Westinghouse $501 \mathrm{G}$, the improvement by a "rule of thumb" will be about $2 / 3$ of the turbine efficiency improvement, the reduction being due to reduced exhaust turbulent heating from turbulence in the diffuser. This would reduce the net plant improvements range from 0.35 to 0.58 percent. Only the upper third of this range would meet the stated goal. Goal compliance is dependent on which baseline governs, an unknown.

Achieving significantly better results than were achieved may first require a more efficient TRF design. Also, at this point, not all flow losses downstream have been addressed. 
Based on these results, CIEA intends to continue researching and improving the lowturbulence strutlet concept. The lower vibration and reduced low frequency noise are also quite important in modern turbine design.

A comment is in order about diffuser mechanical design. We used nearly the same model diffuser wall thickness for small and large diffuser sections. The large sections vibrated more, regardless of other factors. There is a lesson here, applicable to real turbine diffusers. To maintain dynamic similarity, and thus identical vibration velocity levels, wall thickness would have to increase in proportion to the diffuser diameter change. For example, scaling up a successful diffuser design from, say, a $25 \mathrm{MW}$ turbine to $250 \mathrm{MW}$, would, ideally, require an overall diameter increase and wall thickness increase by a factor of 3.16 (square root of the mass flow or power ratio.) Economics, and other technical factors, argue convincingly against this wall thickening. Thus, a large but economical diffuser using the same metal thickness as its smaller predecessor is much more responsive to low frequency flow noise and turbulence, and will thus vibrate more energetically. Metal stresses will be higher, at least in places. So, scaled-up yet economical diffuser designs must be more sophisticated, both mechanically and flow-wise, for similar vibration, noise and stress levels. Thus, the same holds true to keep the same level of maintenance cost and useful life, compared to a smaller turbine diffuser.

\section{Conclusions of Appendix A Work}

1. Both augmented turbulence and the suppressed turbulence strutlet design were effective at increasing the $\mathrm{Cp}$ above the baseline test. The augmented turbulence was a fraction of a $\mathrm{Cp}$ unit better at the time of this writing.

2. The suppressed turbulence diffuser design with strutlets had noticeably and significantly less variation in $\mathrm{Cp}$.

3. The rapid variation in $\mathrm{Cp}$ is indicative of low frequency noise generation potential in the diffuser. A proportion of this measured sound is expected to be radiated out the diffuser outlet. This author believes this to be the case, but the present data fall short of providing a complete proof.

4. The suppressed turbulence design had at least $5 \mathrm{~dB}$ less low-frequency acoustic pressure at its inlet, compared to the augmented turbulence and baseline diffusers.

5. The estimated improvement demonstrated as available in turbine power and heat rate, over both model and actual $\mathrm{Cp}$ baseline, are a range of 0.52 to 0.87 percent simple cycle, which are above the overall project goal of 0.5 percent. Using a rule of thumb, an efficient combined cycle plant will benefit about two-thirds as much, reducing the range to 0.58 to 0.35 percent. Only the first of these numbers is above the stated goal. 
6. Greenhouse gas emissions would be reduced by approximately the same amounts as the efficiency increases, for the same power.

7. The Phase 1 baseline actual turbine test $\mathrm{Cp}$ test values were unlikely to be impacted by more than $0.02 \mathrm{Cp}$ units by any static pressure recovery that might occur in the 10 inches between the probe location and the turbine wheel.

8. Diffuser designs scaled up from smaller turbine diffusers can require more sophisticated flow and mechanical designs for equivalent vibration velocity, and thus, for equivalent maintenance cost and useful life. 